\title{
Crossing symmetry in alpha space
}

\section{Matthijs Hogervorst ${ }^{a}$ and Balt C. van Rees ${ }^{b}$}

${ }^{a}$ C.N. Yang Institute for Theoretical Physics, Stony Brook University, Stony Brook, NY 11794-3840, U.S.A.

${ }^{b}$ Centre for Particle Theory $\&$ Department of Mathematical Sciences, Durham University, South Road, Durham DH1 3LE, U.K.

E-mail: mhogervorst@perimeterinstitute.ca, balt.van-rees@durham.ac.uk

Abstract: We initiate the study of the conformal bootstrap using Sturm-Liouville theory, specializing to four-point functions in one-dimensional CFTs. We do so by decomposing conformal correlators using a basis of eigenfunctions of the Casimir which are labeled by a complex number $\alpha$. This leads to a systematic method for computing conformal block decompositions. Analyzing bootstrap equations in alpha space turns crossing symmetry into an eigenvalue problem for an integral operator $\mathrm{K}$. The operator $\mathrm{K}$ is closely related to the Wilson transform, and some of its eigenfunctions can be found in closed form.

KEywords: Conformal Field Theory, Field Theories in Lower Dimensions

ArXiv EPrint: 1702.08471 


\section{Contents}

1 Introduction 1

2 One-dimensional bootstrap and alpha space 4

2.1 Sturm-Liouville theory of the $\mathrm{SL}(2, \mathbb{R})$ Casimir 4

$\begin{array}{lll}2.2 & \text { Alpha space as a Jacobi transform } & 7\end{array}$

2.3 Convergence of the alpha space transform 9

2.4 Conformal block decomposition 11

2.4.1 Examples 13

$\begin{array}{lll}2.5 & \text { Convergence and asymptotics } & 15\end{array}$

$\begin{array}{ll}\text { 2.5.1 Application: OPE convergence } & 16\end{array}$

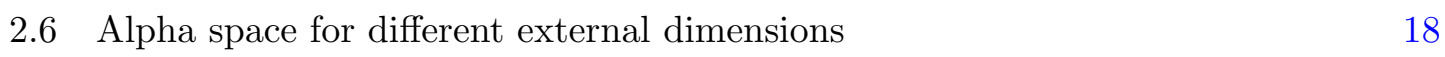

3 Crossing kernel $\quad 20$

3.1 General case 20

$\begin{array}{ll}3.2 & \text { Identical operators } \\ 3.3 & 21\end{array}$

3.3 Functional properties of the crossing kernels 22

3.4 Computation of the crossing kernel 23

$3.5 \mathrm{~K}$ and $\widetilde{\mathrm{K}}$ as intertwiners 24

$\begin{array}{ll}3.6 & \text { Analytic structure of the crossing kernel }\end{array}$

$\begin{array}{lll}3.7 & \text { Symmetries of the crossing kernel } & 27\end{array}$

4 Applications to the conformal bootstrap $\quad 28$

$\begin{array}{lll}4.1 & \text { (Dis)proving a false theorem } & 29\end{array}$

$\begin{array}{lll}4.2 & \text { Split kernel } & 29\end{array}$

4.3 Using the $\xi_{n}$ as a basis 32

5 Discussion $\quad 35$

A Computing the inner product $\left\langle\Psi_{\alpha}, \Psi_{\beta}\right\rangle \quad 36$

\section{Introduction}

Symmetries and consistency conditions play an important role in quantum field theory. This is especially true in the realm of Conformal Field Theories (CFTs), which can be analyzed by combining constraints from conformal invariance, unitarity and crossing symmetry. This set of ideas is known as the conformal bootstrap [1-4]. It was revived in [5] and has led to a wealth of numerical and analytical results about CFTs, see for instance [6-28]. ${ }^{1}$

\footnotetext{
${ }^{1}$ See [29-31] for an introductory discussion of the conformal bootstrap.
} 
Since the bootstrap uses constraints coming from correlation functions, it is natural to express crossing symmetry as a sum rule in position space. This is not strictly necessary: for instance, some properties of CFT correlators are more transparent in Mellin space [32-40]. In the present paper we introduce alpha space, an integral transform for CFT correlators based on the Sturm-Liouville theory of the conformal Casimir operator. As we will explain, alpha space can be used to rephrase crossing symmetry as an eigenvalue problem.

To illustrate this idea, consider the toy crossing equation

$$
\sum_{n=0}^{5} c_{n} p_{n}(z)=\sum_{n=0}^{5} c_{n} p_{n}(1-z)
$$

involving the following polynomials: ${ }^{2}$

$$
p_{n}(z)=(-1)^{n} \sum_{j=0}^{n} 2^{j}\left(\begin{array}{c}
5-z \\
n-j
\end{array}\right)\left(\begin{array}{l}
z \\
j
\end{array}\right), \quad n=0, \ldots, 5 .
$$

How can we determine the set of all $c_{n}$ that satisfy (1.1)? Since the $p_{n}(z)$ are polynomials, various brute-force methods can be used. More elegantly, we can realize that the $p_{n}$ form a complete basis for the space of polynomials of degree $\leq 5$, orthogonal with respect to the inner product

$$
\int d \mu f(z) g(z), \quad \int d \mu=\sum_{k=0}^{5} \frac{(-1)^{k}}{2^{k}}\left(\begin{array}{l}
5 \\
k
\end{array}\right) \int d z \delta(z-k) .
$$

This implies that the $p_{n}(1-z)$ appearing in the r.h.s. of the crossing equation can be decomposed as follows:

$$
p_{n}(1-z)=\sum_{m=0}^{5} \mathrm{Q}_{n}^{m} p_{m}(z)
$$

for some $6 \times 6$ matrix $\mathrm{Q}$. The latter can be easily computed using (1.3). Since $z \mapsto 1-z$ is an involution, we must have $Q^{2}=\mathbb{1}_{6 \times 6}$, as can be checked easily. Eq. (1.1) can now be recast as

$$
c_{n}=(\mathrm{Q} \cdot c)_{n}
$$

hence our problem reduces to finding all eigenvectors of $Q$ with eigenvalue +1 . There are three such eigenvectors:

$$
f_{1}=p_{0}(\equiv 1) \quad f_{2}=2 p_{1}-p_{3}-p_{4}, \quad f_{3}=p_{2}+2 p_{3}+2 p_{4},
$$

so the most general solution to (1.1) is

$$
\sum_{n=0}^{5} c_{n} p_{n}(z)=\sum_{i=1}^{3} t_{i} f_{i}(z), \quad t_{i} \in \mathbb{R} .
$$

\footnotetext{
${ }^{2} \mathrm{Up}$ to a choice of normalization, these are the Kravchuk polynomials with $N=5$ and $p=1 / 2[41,42]$.
} 
In this paper we consider one-dimensional (defect) CFTs which are governed by crossing equations similar to (1.1). For definiteness, let us consider a four-point function $F(z)$ of identical operators of dimension $h_{\phi}$, admitting a conformal block decomposition

$$
F(z)=\int_{0}^{\infty} d h \rho(h) k_{h}(z), \quad \rho(h)=\sum_{n} c_{n} \delta\left(h-h_{n}\right),
$$

where the $k_{h}(z)$ are $\mathrm{SL}(2, \mathbb{R})$ conformal blocks:

$$
k_{h}(z)=z_{2}^{h} F_{1}(h, h ; 2 h ; z) .
$$

The spectrum $\left\{h_{n}\right\}$ and the OPE coefficients $c_{n} \geq 0$ are typically unknown. Bootstrapping entails computing or constraining these CFT data using the crossing relation

$$
F(z)=\left(\frac{z}{1-z}\right)^{2 h_{\phi}} F(1-z) .
$$

There are various technical differences between this $d=1$ bootstrap problem and the previous toy example. For one, $h$ takes its values in the continuum $\mathbb{R}_{\geq 0}$, whereas the toy example had a finite and discrete spectrum. Nevertheless, it is tantalizing to apply the logic from the toy example to the bootstrap. For instance, one could hope to constrain the density $\rho(h)$ from (1.8) through a relation of the form

$$
\rho(h) \stackrel{?}{=} \int_{0}^{\infty} d h^{\prime} \mathscr{Q}\left(h, h^{\prime} \mid h_{\phi}\right) \rho\left(h^{\prime}\right)
$$

for some continuous kernel $\mathscr{Q}\left(h, h^{\prime} \mid h_{\phi}\right)$ which plays the role of Q. Sadly eq. (1.11) cannot quite be true. The reason is that the conformal blocks $k_{h}(z)$ don't form an orthogonal basis of functions on $(0,1)$. The principal aim of this paper is to demonstrate that it is nevertheless possible to write down a qualitatively very similar relation. In order to do so we use a new basis of functions to transform our four-point function to a space that we denote as alpha space. In this space we can properly define (1.11) in terms of a crossing symmetry kernel $K$ which we will explicitly compute. We will discuss its main features and explain how the ordinary conformal block decomposition is recovered from an analytic continuation in alpha.

We stress that the philosophy of studying CFTs using crossing kernels — à la (1.11) is not new. An early avatar of this idea can be found in eq. (2.66) of ref. [43]. Nonetheless, we are not aware of earlier work where the relevant $\mathrm{SO}(d, 2)$ or $\mathrm{SL}(2, \mathbb{R})$ crossing kernels have been worked out in detail. An exception is the $2 d$ Liouville CFT, for which the crossing kernels have been computed [44, 45] as the $6-j$ symbol of a class of representations of $U_{q}(\mathfrak{s l}(2, \mathbb{R}))$, leading to a formal proof of consistency of the theory. ${ }^{3}$ The case of rational $2 d$ CFTs (i.e. Virasoro minimal models) is also of interest, since in such theories the crossing kernel is realized as a finite matrix [49-51]. We will comment on the group-theoretic interpretation of our crossing symmetry kernel in section 5 .

The outline of this paper is as follows. In section 2 we review the one-dimensional bootstrap problem and solve the Sturm-Liouville problem for the $\operatorname{SL}(2, \mathbb{R})$ Casimir operator. This allows us to construct a complete, orthogonal basis of eigenfunctions on the

\footnotetext{
${ }^{3}$ See also $[46-48]$.
} 
interval $(0,1)$. In section 3 we use these basis functions to derive a crossing equation similar to (1.11), and we study the properties of the relevant kernel $K$. Section 4 describes several possible applications of crossing kernels to the conformal bootstrap.

Note added: while preparing this manuscript we learned about ref. [52], which discusses a crossing kernel approach to both $\mathrm{SU}(2)$ and conformal crossing symmetry equations and is tangentially related to this paper.

\section{One-dimensional bootstrap and alpha space}

This section is devoted to the Sturm-Liouville theory of the conformal Casimir of $\operatorname{SL}(2, \mathbb{R})$, the conformal group in one spacetime dimension. One-dimensional CFTs arise in the description of line defects in higher-dimensional theories [53-56]. Although 1d CFTs are in many ways more tractable than $d$-dimensional systems, we also note that many salient features of the $d$-dimensional bootstrap already appear at the level of $d=1$. In addition the $1 d$ conformal blocks appear naturally in the light-cone limit of the higher-dimensional crossing symmetry equations, where it becomes possible to obtain non-trivial analytic results $[12,13,57]$.

\subsection{Sturm-Liouville theory of the $\operatorname{SL}(2, \mathbb{R})$ Casimir}

We will start by analyzing the four-point function of a single primary (or lowest-weight) operator $\phi(x)$ in a $1 d$ CFT. The general case will be addressed in section 2.6. The only quantum number of $\phi$ is its scaling dimension $h_{\phi}$, and conformal symmetry dictates that $\langle\phi \phi \phi \phi\rangle$ has the following form:

$$
\left\langle\phi\left(x_{1}\right) \phi\left(x_{2}\right) \phi\left(x_{3}\right) \phi\left(x_{4}\right)\right\rangle=\frac{F_{\phi \phi \phi \phi}(z)}{\left|x_{1}-x_{2}\right|^{2 h_{\phi}}\left|x_{3}-x_{4}\right|^{2 h_{\phi}}}
$$

where the points $x_{i} \in \mathbb{R}$ lie on a line and $z$ is the following cross ratio:

$$
z:=\frac{\left|x_{12}\right|\left|x_{34}\right|}{\left|x_{13}\right|\left|x_{24}\right|} \in(0,1)
$$

writing $x_{i j}=x_{i}-x_{j} .{ }^{4}$ The function $F_{\phi \phi \phi \phi}(z)$ admits the following conformal block (CB) decomposition:

$$
F_{\phi \phi \phi \phi}(z)=\sum_{\mathcal{O}} \lambda_{\phi \phi \mathcal{O}}^{2} k_{h_{\mathcal{O}}}(z)
$$

where the functions $k_{h}(z)$ are the $1 d$ conformal blocks defined in eq. (1.9). The sum runs over all operators $\mathcal{O}$ in the $\phi \times \phi$ OPE of dimension $h_{\mathcal{O}}$, and $\lambda_{\phi \phi \mathcal{O}}$ is the $\mathcal{O} \in \phi \times \phi$ OPE coefficient. Finally, crossing symmetry (invariance under the exchange $x_{i} \leftrightarrow x_{j}$ ) of the $\langle\phi \phi \phi \phi\rangle$ correlator leads to the bootstrap constraint

$$
F_{\phi \phi \phi \phi}(z)=\left(\frac{z}{1-z}\right)^{2 h_{\phi}} F_{\phi \phi \phi \phi}(1-z)
$$

which must hold for all $0 \leq z \leq 1$.

\footnotetext{
${ }^{4}$ Although a priori the variable $z$ is not restricted to the unit interval, we require $z \in(0,1)$ to guarantee OPE convergence on both sides of the bootstrap equation.
} 
We will not assume unitarity (i.e. reflection positivity) in this paper. Just for completeness, we recall that if the CFT in question is unitary, the decomposition (2.3) is constrained as follows:

- the $\lambda_{\phi \phi \mathcal{O}}$ must be real-valued, hence $\lambda_{\phi \phi \mathcal{O}}^{2}>0$;

- there must be a contribution of the unit operator $\mathbb{1}$ with $h_{\mathbb{1}}=0$ and $\lambda_{\phi \phi \mathbb{1}}=1$;

- all other operators (including $\phi$ ) have $h_{\mathcal{O}}>0$.

As noted in the introduction, it is conventional in the CFT literature to investigate the bootstrap equation (2.4) in position space. Here we will take a different approach. We start by remarking that the conformal blocks $k_{h}(z)$ are eigenfunctions of a second-order differential operator $D$, the quadratic Casimir operator of $\operatorname{SL}(2, \mathbb{R})$ :

$$
D \cdot k_{h}(z)=h(h-1) k_{h}(z), \quad D=z^{2}(1-z) \partial^{2}-z^{2} \partial .
$$

In what follows, we will develop the Sturm-Liouville theory of the operator $D$ on the interval $(0,1) .{ }^{5}$ As a first step, we notice that $D$ can be written in the following suggestive form:

$$
D \cdot f(z)=z^{2} \frac{\partial}{\partial z}\left[(1-z) f^{\prime}(z)\right]
$$

This implies that $D$ is self-adjoint with respect to the inner product

$$
\langle f, g\rangle=\int_{0}^{1} \frac{d z}{z^{2}} \overline{f(z)} g(z)
$$

where $f, g$ are functions $(0,1) \rightarrow \mathbb{C}$ that are well-behaved near $z=0$ and $z=1$. Indeed, we have

$$
\langle f, D \cdot g\rangle-\langle D \cdot f, g\rangle=\int_{0}^{1} d z \frac{\partial}{\partial z}\left[(1-z)\left(\bar{f} g^{\prime}-\overline{f^{\prime}} g\right)\right]
$$

which is a boundary term. Of course, not all functions have a finite norm with respect to the inner product (2.7). Requiring that a function $f$ is square integrable leads to the following constraints on its asymptotics near $z=0$ and $z=1$ :

$$
f(z) \underset{z \rightarrow 0}{\sim} z^{1 / 2+\epsilon} \quad \text { and } \quad f(z) \underset{z \rightarrow 1}{\sim}(1-z)^{-1 / 2+\epsilon^{\prime}}
$$

for constants $\epsilon, \epsilon^{\prime}>0$. In particular, this implies that in a unitary CFT all four-point functions $F_{\phi \phi \phi \phi}(z)$ have a divergent norm with respect to (2.7).

Our next order of business is to construct an orthogonal basis of eigenfunctions of $D$. We start by solving the eigenvalue equation $D \cdot f=\lambda f$. After writing $\lambda=\alpha^{2}-1 / 4$ for convenience, we find that the general solution (for $\alpha \neq 0$ ) is given by

$$
f(z)=A_{1}(\alpha) k_{\frac{1}{2}+\alpha}(z)+A_{2}(\alpha) k_{\frac{1}{2}-\alpha}(z)
$$

\footnotetext{
${ }^{5}$ Note added: although we have not attempted to do so, it is in principle possible to change the boundary conditions at $z=1$ [58]. We thank Miguel Paulos for pointing out this reference.
} 
for two constants $A_{1,2}(\alpha)$ that are to be determined. In order to fix them, let's analyze the $z \rightarrow 0,1$ asymptotics of $f(z)$. First, we notice that the blocks themselves are logarithmically divergent near $z=1$. To be precise, we have

$$
k_{\frac{1}{2}+\alpha}(z) \underset{z \rightarrow 1}{\sim}-\frac{\Gamma(1+2 \alpha)}{\Gamma^{2}\left(\frac{1}{2}+\alpha\right)} \ln (1-z)+\text { regular }
$$

and likewise for $k_{\frac{1}{2}-\alpha}(z)$. Requiring that (2.10) has a finite limit as $z \rightarrow 1$ therefore determines the relative coefficient $A_{1}(\alpha) / A_{2}(\alpha)$. Fixing the overall normalization by imposing $f(1)=1$, we arrive at the following eigenfunctions: ${ }^{6}$

$$
\Psi_{\alpha}(z)=\frac{1}{2}\left[Q(\alpha) k_{\frac{1}{2}+\alpha}(z)+Q(-\alpha) k_{\frac{1}{2}-\alpha}(z)\right], \quad Q(\alpha)=\frac{2 \Gamma(-2 \alpha)}{\Gamma^{2}\left(\frac{1}{2}-\alpha\right)} .
$$

In what follows, it will be useful to rewrite $\Psi_{\alpha}(z)$ as

$$
\Psi_{\alpha}(z)={ }_{2} F_{1}\left(\begin{array}{c}
\frac{1}{2}+\alpha, \frac{1}{2}-\alpha \\
1
\end{array} ; \frac{z-1}{z}\right)
$$

using a hypergeometric identity. In particular, this makes it manifest that $\Psi_{\alpha}(1)=1$. However, we have not yet inspected the asymptotics near $z=0$. Assuming that $\alpha$ is real, we find that $\Psi_{\alpha}(z) \underset{z \rightarrow 0}{\sim} z^{1 / 2-|\alpha|}$, which means that the functions $\Psi_{\alpha}$ have infinite norm. The only way to avoid this problem is to assume that $\alpha$ is imaginary. In that case, we find that $\Psi_{\alpha}$ has the following asymptotics:

$$
\Psi_{\alpha}(z) \underset{z \rightarrow 0}{\sim}|Q(\alpha)| \sqrt{z} \cos (\operatorname{Im}(\alpha) \ln z+\text { const. }) \quad[\alpha \in i \mathbb{R}]
$$

implying that $\Psi_{\alpha}$ is rapidly oscillating near $z=0$. Notice that even for imaginary $\alpha$, the function $\Psi_{\alpha}(z)$ is real-valued, since it is symmetric under $\alpha \rightarrow-\alpha$. A plot of two different functions $\Psi_{\alpha}(z)$ is shown in figure 1 .

Since $\Psi_{\alpha}(z)$ oscillates near $z=0$ at a rate that depends on $\alpha$, it is at least plausible that $\left\langle\Psi_{\alpha}, \Psi_{\beta}\right\rangle=0$ for $\alpha \neq \pm \beta$, cf. the Fourier transform on $\mathbb{R}$. This is confirmed by an explicit computation, performed in appendix A. There it is shown that the inner product $\left\langle\Psi_{\alpha}, \Psi_{\beta}\right\rangle$ behaves as a delta function on the imaginary axis. To be precise: if $f(\alpha)$ is defined on $i \mathbb{R}$ and has compact support, we have

$$
\frac{1}{2 \pi i} \int_{-i \infty}^{i \infty} d \beta\left\langle\Psi_{\alpha}, \Psi_{\beta}\right\rangle f(\beta)=N(\alpha) \frac{f(\alpha)+f(-\alpha)}{2}
$$

where

$$
N(\alpha)=\frac{\Gamma(\alpha) \Gamma(-\alpha)}{2 \pi \Gamma\left(\frac{1}{2}+\alpha\right) \Gamma\left(\frac{1}{2}-\alpha\right)}=\frac{|Q(\alpha)|^{2}}{2} \geq 0 .
$$

Informally, eq. (2.15) shows that the functions $\Psi_{\alpha}(z)$ are plane-wave normalized, having norm $N(\alpha)$. The fact that the r.h.s. of (2.15) contains the sum $\frac{1}{2}[f(\alpha)+f(-\alpha)]$ reflects that $\Psi_{\alpha}$ is even in $\alpha$, which carries over to the inner product $\left\langle\Psi_{\alpha}, \Psi_{\beta}\right\rangle$.

\footnotetext{
${ }^{6}$ Remarkably, these are not the usual 'shadow-symmetric' blocks obtained by integrating one-dimensional three-point functions over the real axis [59]. Indeed, in one dimension this integral is easily performed using the techniques of [60] and diverges logarithmically as $z \rightarrow 1$, in contrast with our $\Psi_{\alpha}(z)$.
} 


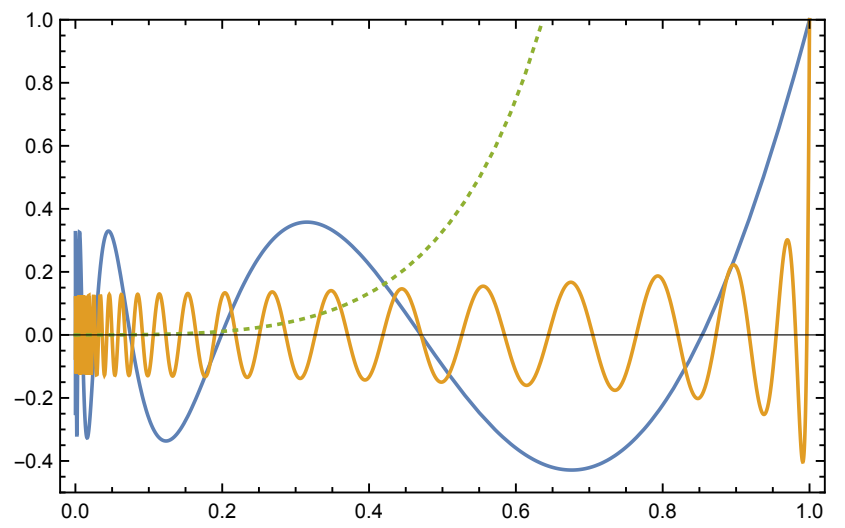

Figure 1. Plot of $z^{-1 / 2} \Psi_{\alpha}(z)$ for $\alpha=2 i$ (blue) and $\alpha=20 i$ (orange), as well as $k_{h}(z)$ for $h=3$ (dotted green). Both the oscillatory behaviour of the $\Psi_{\alpha}(z)$ near $z=0$ and their $O(\sqrt{z})$ growth are clearly visible.

Summarizing, we have constructed a set of orthogonal eigenfunctions $\Psi_{\alpha}(z)$ with respect to the inner product (2.7). Naively, we can appeal to familiar arguments of SturmLiouville theory to argue that these eigenfunctions form a complete set. In other words, we can decompose a given function $f:(0,1) \rightarrow \mathbb{R}$ as follows:

$$
f(z)=\frac{1}{2 \pi i} \int_{-i \infty}^{i \infty} \frac{d \alpha}{N(\alpha)} \widehat{f}(\alpha) \Psi_{\alpha}(z) \Leftrightarrow \widehat{f}(\alpha)=\int_{0}^{1} \frac{d z}{z^{2}} f(z) \Psi_{\alpha}(z)
$$

This formula describes how $f(z)$ is encoded by its "spectral density" $\widehat{f}(\alpha)$, and vice versa. A mathematically rigorous way to obtain this identity will be described in the next section.

A sufficient condition for eq. (2.17) to make sense is that $f$ be square integrable:

$$
\langle f, f\rangle=\int_{0}^{1} \frac{d z}{z^{2}}|f(z)|^{2}<\infty .
$$

An equivalent condition (see the next section) is that

$$
\frac{1}{2 \pi i} \int_{-i \infty}^{i \infty} \frac{d \alpha}{N(\alpha)}|\widehat{f}(\alpha)|^{2}
$$

is finite. In sections 2.4 and 2.5 we discuss how these constraints can be loosened.

Eq. (2.15) shows that the $\Psi_{\alpha}(z)$ form a complete basis in $\alpha$ space. For reference, we remark that the $\Psi_{\alpha}(z)$ also obey a completeness relation in position space, namely

$$
\frac{1}{2 \pi i} \int_{-i \infty}^{i \infty} \frac{d \alpha}{N(\alpha)} \Psi_{\alpha}(z) \Psi_{\alpha}(w)=z^{2} \delta(z-w)
$$

as can be deduced from (2.17).

\subsection{Alpha space as a Jacobi transform}

The alpha space transform $f(z) \mapsto \widehat{f}(\alpha)$ is closely related to a known integral transform, known as the Jacobi transform. We will briefly describe this transform in the rest of this 
section, pointing to refs. [61-63] as a point of entry in the mathematics literature. The Jacobi transform is an integral transform that makes use of the Jacobi functions:

$$
\vartheta_{\alpha}^{(p, q)}(x):={ }_{2} F_{1}\left(\begin{array}{c}
\frac{1}{2}(1+p+q)+\alpha, \frac{1}{2}(1+p+q)-\alpha \\
p+1
\end{array} ;-x\right), \quad x \geq 0 .
$$

The parameters $p, q \geq 0$ are fixed, whereas the label $\alpha \in i \mathbb{R}$ is allowed to vary continuously. Notice that $\vartheta_{\alpha}^{(p, q)}(x)$ is even in $\alpha$, and therefore real-valued. Consider now a complex function $f(x)$, defined for $x \geq 0$, decaying sufficiently fast as $x \rightarrow \infty$. We assign to it its Jacobi transform $\mathcal{J} f$ as follows:

$$
f(x) \mapsto(\mathcal{J} f)(\alpha):=\int_{0}^{\infty} d x \omega_{p, q}(x) f(x) \vartheta_{\alpha}^{(p, q)}(x), \quad \omega_{p, q}(x)=x^{p}(1+x)^{q} .
$$

$\omega_{p, q}(x)$ plays the role of a weight function in position space. A standard result - see Theorem 2.3 of ref. [63] — is that $f$ can be restored from its Jacobi transform:

$$
f(x)=\frac{1}{2 \pi i} \int_{-i \infty}^{i \infty} \frac{d \alpha}{\mathscr{N}_{p, q}(\alpha)}(\mathcal{J} f)(\alpha) \vartheta_{\alpha}^{(p, q)}(x)+\ldots
$$

where

$$
\mathscr{N}_{p, q}(\alpha)=\frac{2 \Gamma^{2}(1+p) \Gamma( \pm 2 \alpha)}{\Gamma\left(\frac{1}{2}(1+p+q) \pm \alpha\right) \Gamma\left(\frac{1}{2}(1+p-q) \pm \alpha\right)}, \quad \Gamma(x \pm y):=\Gamma(x+y) \Gamma(x-y) .
$$

The dots in (2.23) indicate that depending on the values of $p$ and $q$ a finite number of terms must be added; equivalently, the integration contour in $\alpha$ can be deformed to pick up poles coming from $1 / \mathscr{N}_{p, q}(\alpha){ }^{7}$

Properly speaking, $\mathcal{J}$ furnishes a map from the Hilbert space $L^{2}\left(\mathbb{R}_{+}, \omega_{p, q}(x) d x\right)$ to the space of functions on $i \mathbb{R}$ which are normalizable with respect to the measure $d \alpha / \mathscr{N}_{p, q}(\alpha)$. This map is an isometry: given two complex functions $f, g$, the following Parseval formula holds:

$$
\int_{0}^{\infty} d x \omega_{p, q}(x) \overline{f(x)} g(x)=\frac{1}{2 \pi i} \int_{-i \infty}^{i \infty} \frac{d \alpha}{\mathscr{N}_{p, q}(\alpha)} \overline{(\mathcal{J} f)(\alpha)}(\mathcal{J} g)(\alpha) .
$$

Specializing to the case $f=g$, this shows in which sense the Jacobi transform is unitary.

It is now straightforward to see that the alpha space transform for the $\operatorname{SL}(2, \mathbb{R})$ Casimir is a special case of the Jacobi transform with $p=q=0$, after the change of variable $x \rightarrow(1-z) / z$. The precise dictionary is given by

$$
\Psi_{\alpha}(z)=\vartheta_{\alpha}^{(0,0)}\left(\frac{1-z}{z}\right), \quad \int_{0}^{\infty} d x \omega_{0,0}(x)=\int_{0}^{1} \frac{d z}{z^{2}}, \quad N(\alpha)=\mathscr{N}_{0,0}(\alpha) .
$$

A direct consequence is the identity

$$
\langle f, g\rangle=\frac{1}{2 \pi i} \int_{-i \infty}^{i \infty} \frac{d \alpha}{N(\alpha)} \bar{f}(\alpha) \widehat{g}(\alpha) .
$$

\footnotetext{
${ }^{7} \mathrm{~A}$ sufficient condition for such terms to be absent is $p+q+1>0$ and $p-q+1>0$.
} 
It would be interesting to see if further theorems concerning the Jacobi transform can be recycled to prove results about alpha space densities in CFTs.

Our discussion has been quite abstract so far and at this stage the reader may want to experiment with some explicit alpha space computations. To do so, it is useful to know that the Jacobi transform essentially maps rational functions to polynomials. A precise statement is the following. Let

$$
P_{n}^{(p, q)}(x)=\frac{(p+1)_{n}}{n !}{ }_{2} F_{1}\left(-n, n+p+q+1 ; p+1 ; \frac{1}{2}(1-x)\right)
$$

be a Jacobi polynomial of degree $n$. Then for any $r, s \geq 0$ we have [64]

$$
\begin{aligned}
\int_{0}^{\infty} d x \omega_{p, q}(x) & \frac{1}{(1+x)^{\frac{1}{2}(p+q+r+s)+1}} P_{n}^{(p, r)}\left(\frac{1-x}{1+x}\right) \vartheta_{\alpha}^{(p, q)}(x) \\
= & \frac{(-1)^{n}}{n !} \frac{\Gamma(p+1) \Gamma\left(\frac{1}{2}(r+s+1) \pm \alpha\right)}{\Gamma\left(\frac{1}{2}(p+q+r+s)+1+n\right) \Gamma\left(\frac{1}{2}(p-q+r+s)+1+n\right)} \\
& \times \mathfrak{p}_{n}\left(\alpha ; \frac{1}{2}(p+q+1), \frac{1}{2}(p-q+1), \frac{1}{2}(r+s+1), \frac{1}{2}(r-s+1)\right) .
\end{aligned}
$$

The object on the last line is a Wilson polynomial $[41,42,65]$ :

$\mathfrak{p}_{n}(\alpha ; a, b, c, d)=(a+b)_{n}(a+c)_{n}(a+d)_{n} F_{3}\left(\begin{array}{c}-n, a+\alpha, a-\alpha, n+a+b+c+d-1 \\ a+b, a+c, a+d\end{array} ; 1\right)$.

Evidently $\mathfrak{p}_{n}(\alpha ; a, b, c, d)$ is a polynomial of degree $n$ in $\alpha^{2}$, and it can be shown that $\mathfrak{p}_{n}$ depends symmetrically on its parameters $a, b, c, d$. Specializing to alpha space $(p=q=0)$ whilst setting $r \rightarrow 0, s \rightarrow 2 \rho-2$, the identity (2.29) becomes

$$
\int_{0}^{1} \frac{d z}{z^{2}} z^{\rho} P_{n}(2 z-1) \Psi_{\alpha}(z)=\frac{(-1)^{n}}{n !} \frac{\Gamma\left(\rho-\frac{1}{2} \pm \alpha\right)}{\Gamma^{2}(\rho+n)} \mathfrak{p}_{n}\left(\alpha ; \frac{1}{2}, \frac{1}{2}, \rho-\frac{1}{2}, \frac{3}{2}-\rho\right)
$$

where $P_{n}$ is a Legendre polynomial of degree $n$. This formula can be used to find the alpha space counterpart of rather general functions in position space. As a simple example, we can set $n=0$ to find the alpha space version of the function $z \mapsto z^{\rho}$ :

$$
\int_{0}^{1} \frac{d z}{z^{2}} z^{\rho} \Psi_{\alpha}(z)=\frac{\Gamma\left(\rho-\frac{1}{2} \pm \alpha\right)}{\Gamma^{2}(\rho)} .
$$

An additional example will be discussed in section 2.4.1.

\subsection{Convergence of the alpha space transform}

Before we turn to the application of alpha space to CFTs, let us comment on the convergence of the alpha space transform $f(z) \mapsto \widehat{f}(\alpha)$. We have in mind a function $f(z)$ that has power-law growth at $z=0$ and $z=1$, i.e.

$$
f(z) \underset{z \rightarrow 0}{\sim} z^{p} \quad \text { and } \quad f(z) \underset{z \rightarrow 1}{\sim} \frac{1}{(1-z)^{q}} .
$$


Moreover, we assume that $f(z)$ admits an expansion in powers of $z^{h}$ around $z=0$, meaning that it is possible to write $f(z)=\sum_{n=1}^{\infty} c_{n} z^{h_{n}}$. All of these conditions are certainly satisfied when $f(z)$ describes a CFT correlation function.

Let us first consider the case where $p>\frac{1}{2}$ and $q<1$. In that case, the integral defining its alpha space density

$$
\widehat{f}(\alpha):=\int_{0}^{1} \frac{d z}{z^{2}} f(z) \Psi_{\alpha}(z)
$$

converges whenever $|\Re(\alpha)|<p-\frac{1}{2}$, meaning that $\widehat{f}(\alpha)$ is holomorphic on a finite strip. Moreover, using the alpha space transform of a single power law (2.32), it is possible to show that $\widehat{f}(\alpha)$ extends to a meromorphic function on the entire complex plane, with poles at $\alpha=h_{n}-\frac{1}{2}+\mathbb{N}$ for $n=1,2, \ldots$ (plus mirror poles on the left half plane).

Next, consider the case $p<\frac{1}{2}, q<1$. In this case, it is convenient to decompose $f(z)$ as

$$
f(z)=f_{\operatorname{sing}}(z)+f_{\text {reg }}(z)
$$

where

$$
f_{\text {sing }}(z)=\sum_{h_{n}<1 / 2} c_{n} z^{h_{n}} \text { and } f_{\mathrm{reg}}(z)=\sum_{h_{n}>1 / 2} c_{n} z^{h_{n}}
$$

By construction, the regular piece $f_{\text {reg }}(z)$ has a well-defined alpha space transform that extends to a meromorphic function on $\mathbb{C}$. We can define the density $\widehat{f}_{\text {sing }}(\alpha)$ termwise, by analytically continuing eq. (2.32) to arbitrary values of $\rho .^{8}$ Concretely, we take the alpha space transform of $f(z)$ to be

$$
\widehat{f}(\alpha)=\sum_{h_{n}<1 / 2} c_{n} \frac{\Gamma\left(h_{n}-\frac{1}{2} \pm \alpha\right)}{\Gamma^{2}\left(h_{n}\right)}+\widehat{f}_{\mathrm{reg}}(\alpha) .
$$

If the leading term of $f_{\operatorname{sing}}(z)$ is a constant, the above argument breaks down, since $1 / \Gamma^{2}(h)$ vanishes when $h \rightarrow 0$. This is an order-of-limits issue, which can be avoided by writing 1 as the limit of $z^{\epsilon}$ as $\epsilon \rightarrow 0$.

Finally, we consider the case $q>1$. For simplicity we consider $p>\frac{1}{2}$, but the case of general $p$ is straightforward to treat using the above discussion. Given that $q>1$, the integral defining $\widehat{f}(\alpha)$ diverges for all values of $\alpha$. We thus regulate this integral by cutting it off at $z=1-\epsilon$, writing

$$
\widehat{f}_{\epsilon}(\alpha):=\int_{0}^{1-\epsilon} \frac{d z}{z^{2}} f(z) \Psi_{\alpha}(z)
$$

Notice that this regulator does not affect the analytic structure of $\widehat{f}(\alpha)$ : all poles originate from the region of integration near $z=0$. Now, to isolate divergent pieces in $\epsilon$ we notice that $\Psi_{\alpha}(z)$ admits an expansion in powers of $(1-z)$ of the following form:

$$
\Psi_{\alpha}(z)=\sum_{k=0}^{\infty} s_{k}(\alpha)(1-z)^{k}
$$

\footnotetext{
${ }^{8}$ Such analytic continuations may require a deformation of the alpha space integration contour away from the imaginary axis. Below we explain how to deal with such cases.
} 
where $s_{k}(\alpha)$ is a polynomial of degree $k$ in $\alpha^{2}$. This implies that $\widehat{f}_{\epsilon}(\alpha)$ has the following structure of divergences: ${ }^{9}$

$$
\widehat{f_{\epsilon}}(\alpha)=[\text { finite as } \epsilon \rightarrow 0]+\sum_{j=0}^{\lfloor q-1\rfloor} \frac{t_{j}(\alpha)}{\epsilon^{q-1-j}}
$$

where $t_{j}(\alpha)$ is a polynomial in $\alpha$. Consequently, we take $\widehat{f}(\alpha)$ to be the finite piece of $\widehat{f}_{\epsilon}(\alpha)$ obtained by subtracting the divergent terms in (2.40).

\subsection{Conformal block decomposition}

As a first application of the alpha space formalism of the previous sections, we will show that it can be used to compute conformal block decompositions for CFT correlators. As a starting point, we have in mind a meromorphic spectral density $\widehat{f}(\alpha)$, even in $\alpha$, written in the following form:

$$
\widehat{f}(\alpha)=\sum_{n} \frac{-R_{n}}{\alpha-\alpha_{n}}+(\alpha \rightarrow-\alpha)+\text { entire. }
$$

The minus sign in front of $R_{n}$ is a choice of convention. We will assume that all poles $\alpha_{n}$ lie on the positive real axis; in particular, we see that every pole has a corresponding mirror pole $-\alpha_{n}$ on the negative real axis.

Our goal is to compute the position space counterpart of $\widehat{f}(\alpha)$ :

$$
f(z)=\int_{\mathcal{C}} \frac{[d \alpha]}{N(\alpha)} \widehat{f}(\alpha) \Psi_{\alpha}(z)
$$

where $\mathcal{C}$ is a contour parallel to the imaginary axis. Here and in what follows we write contour integrals as

$$
\int[d \alpha]=\int_{-i \infty}^{i \infty} \frac{d \alpha}{2 \pi i}
$$

to avoid notational clutter. Notice that both $\widehat{f}(\alpha)$ and the measure $N(\alpha)$ are even in $\alpha$, which means that we can replace $\Psi_{\alpha}(z)$ by any linear combination of the conformal block $Q(\alpha) k_{\frac{1}{2}+\alpha}(z)$ and its shadow $Q(-\alpha) k_{\frac{1}{2}-\alpha}(z)$. Without loss of generality, let us attempt to close the contour $\mathcal{C}$ to the right, picking up all poles $\alpha_{n}$ on the right half plane. This means that we have to drop the shadow part $\sim Q(-\alpha) / N(\alpha) \times k_{\frac{1}{2}-\alpha}(z)$, as it grows

\footnotetext{
${ }^{9}$ To derive this formula, we are assuming that $f(z)$ admits an expansion of the form

$$
f(z)=\frac{1}{(1-z)^{q}}\left[\text { const. }+\sum_{n \geq 1} a_{n}(1-z)^{n}\right]
$$

around $z=1$. If $f(z)$ rather behaves as a more general sum of power laws

$$
f(z)=\frac{c_{1}}{(1-z)^{q_{1}}}+\frac{c_{2}}{(1-z)^{q_{2}}}+\ldots
$$
}

Eq. (2.40) is modified in a straightforward fashion. 


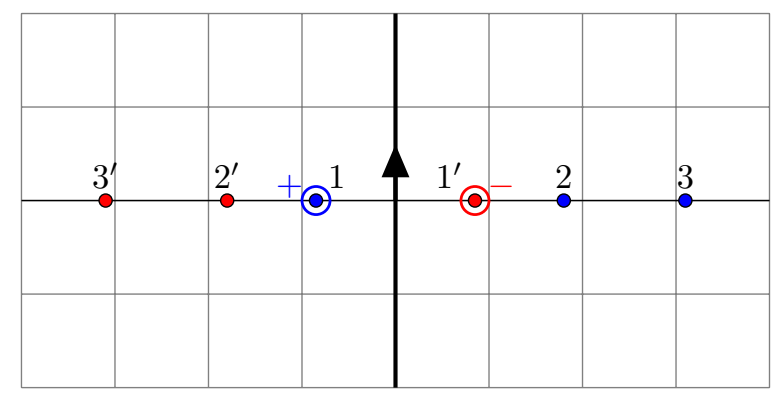

Figure 2. Choice of contour for a typical CFT correlator in the complex $\alpha$-plane. The blue dots, labeled by $\{1,2,3\}$, correspond to physical poles of $\widehat{f}(\alpha)$, whereas their mirrors (in red, with primed labels) are unphysical. The pole 1 has $\Re(\alpha)<0$, hence it corresponds to an operator of dimension $h<1 / 2$. The contour runs upwards along the imaginary axis, but in this case it must circle 1 in the positive and $1^{\prime}$ in the negative direction, as indicated.

exponentially on the right half plane, whereas the conformal block part decreases as $\Re(\alpha) \rightarrow$ $\infty$. Consequently, we find that the position space version of $\widehat{f}(\alpha)$ is given by

$$
f(z)=\int_{\mathcal{C}}[d \alpha] \widehat{f}(\alpha) \frac{Q(\alpha)}{N(\alpha)} k_{\frac{1}{2}+\alpha}(z)=\int_{\mathcal{C}}[d \alpha] \widehat{f}(\alpha) \frac{2}{Q(-\alpha)} k_{\frac{1}{2}+\alpha}(z)
$$

using the second equality in (2.16). In that case, we can rewrite $f(z)$ as

$$
f(z)=\sum_{n} \frac{2 R_{n}}{Q\left(-\alpha_{n}\right)} k_{\frac{1}{2}+\alpha_{n}}(z) .
$$

To pass from eq. (2.44) to (2.45), we used that $1 / Q(-\alpha)$ is analytic on the right half plane. But the sum appearing in the r.h.s. is precisely a CB decomposition - cf. eq. (2.3) - where the $n$-th term corresponds to an exchanged operator $\mathcal{O}_{n}$ of dimension $\left[\mathcal{O}_{n}\right]=1 / 2+\alpha_{n}$, having OPE coefficient

$$
\lambda_{\phi \phi \mathcal{O}_{n}}^{2}=\frac{2 R_{n}}{Q\left(-\alpha_{n}\right)} .
$$

Since $Q(-\alpha)>0$ for all $\alpha>0$, we conclude that $\lambda_{\phi \phi \mathcal{O}_{n}}^{2}$ is positive iff $R_{n}$ is positive.

Above, we assumed that all $\alpha_{n}$ were positive. This means that only operators of dimension $\left[\mathcal{O}_{n}\right]>1 / 2$ appear in the $\mathrm{CB}$ decomposition (2.45). This condition can be loosened: an operator of dimension $h<1 / 2$ would simply correspond to a pole $\alpha_{*}$ lying on the left half plane. We must in this case deform the contour to circle $\alpha_{*}$ in the positive direction. Moreover, $\alpha_{*}$ will have a mirror pole $-\alpha_{*}$ on the right half plane, which must be circled in the negative direction, such that it does not give an anomalous contribution to $f(z)$ - see figure 2. We will revisit this point in section 2.5.

The cases $h=0$ (corresponding to the unit operator) and $h=1 / 2$ require special attention. As for $h=0$, notice that $1 / Q(-\alpha)$ has a pole at $\alpha=-1 / 2$, namely

$$
\frac{1}{Q(-\alpha)} \underset{\alpha \rightarrow-1 / 2}{\sim}-\frac{1}{\alpha+1 / 2}
$$


Consequently, it suffices for $\widehat{f}(\alpha)$ to be finite at $\alpha=1 / 2$ in order to generate a unit operator term. To be precise, if

$$
\widehat{f}(\alpha) \underset{\alpha \rightarrow-1 / 2}{\sim} c+O\left[\left(\alpha+\frac{1}{2}\right)\right]
$$

and the contour is such that it wraps around $\alpha=-1 / 2$ in the sense described above, then $f(z)=2 c+$ other conformal blocks. A similar issue arises if $h=1 / 2$, because $1 / Q(-\alpha)$ vanishes as $\alpha \rightarrow 0$. More precisely

$$
\frac{1}{Q(-\alpha)} \underset{\alpha \rightarrow 0}{\sim} \pi \alpha+O\left(\alpha^{2}\right)
$$

hence in order to obtain a contribution $f(z) \sim c k_{1 / 2}(z) \sim c \sqrt{z}+\ldots$ in position space, we must have

$$
\widehat{f}(\alpha)=-\frac{c}{2 \pi \alpha^{2}}+O\left(\alpha^{-1}\right)
$$

\subsubsection{Examples}

To develop some familiarity with the alpha space representation of correlation functions, we will compute the alpha space transform of some simple functions in $z$-space, and we use these results to compute the resulting conformal block decompositions.

- Let's compute the alpha space transform of a single conformal block $k_{h}(z)$ with $h>1 / 2$ :

$$
\widehat{k_{h}}(\alpha)=\int_{0}^{1} \frac{d z}{z^{2}} k_{h}(z) \Psi_{\alpha}(z)=\frac{\mathscr{C}(h)}{\alpha^{2}-\left(h-\frac{1}{2}\right)^{2}}, \quad \mathscr{C}(h)=-\frac{\Gamma(2 h)}{\Gamma^{2}(h)} .
$$

In order to derive this result, it's convenient to use the Mellin-Barnes formula

$$
{ }_{2} F_{1}(a, b ; c ; z)=\frac{\Gamma(c)}{\Gamma(a) \Gamma(b)} \int_{-i \infty}^{i \infty} d s \frac{\Gamma(-s) \Gamma(a+s) \Gamma(b+s)}{\Gamma(c+s)}(-z)^{s}
$$

in order to expand both $k_{h}(z)$ and $\Psi_{\alpha}(z)$. Alternatively, eq. (2.51) is easy to check numerically inside the strip $|\Re(\alpha)|<h-\frac{1}{2}$.

Let us make two comments about the formula (2.51). First, although the integral in (2.51) converges only in a finite strip, the r.h.s. defines an analytic continuation to any value of $\alpha$. Moreover, the same formula defines an analytic continuation to values of $h<1 / 2$. Second, $\widehat{k_{h}}(\alpha)$ has precisely one pole on the right half plane, at $\alpha=h-1 / 2$, in accordance with our discussion from the previous section.

- Let $f_{p}(z)=z^{p}$ with $p>1 / 2$. We have already encountered this function in eq. (2.32), finding that in alpha space it becomes

$$
\widehat{f}_{p}(\alpha)=\frac{\Gamma\left(p-\frac{1}{2} \pm \alpha\right)}{\Gamma^{2}(p)} .
$$

Let's use this to obtain the CB decomposition of $f_{p}(z)$. First, we note that $\widehat{f}_{p}(\alpha)$ has poles at

$$
\alpha_{n}=p-\frac{1}{2}+n \quad \text { and } \quad \tilde{\alpha}_{n}=\frac{1}{2}-p-n \quad \text { with } \quad n \in \mathbb{N} .
$$


Closing the contour to the right, we only pick up the $\alpha_{n}$ poles. The residue of the $n$-th pole is

$$
R_{n}=-\left.\operatorname{Res} \widehat{f}(\alpha)\right|_{\alpha=\alpha_{n}}=\frac{(-1)^{n}}{n !} \frac{\Gamma(2 p-1+n)}{\Gamma^{2}(p)}
$$

and this pole corresponds to an operator of dimension $h=1 / 2+\alpha_{n}=p+n$. Using the argument from the previous section, we conclude that

$$
f_{p}(z)=\sum_{n=0}^{\infty} \frac{2 R_{n}}{Q\left(-\alpha_{n}\right)} k_{p+n}(z)=\sum_{n=0}^{\infty} \frac{(-1)^{n}}{n !} \frac{(p)_{n}^{2}}{(2 p-1+n)_{n}} k_{p+n}(z) .
$$

This confirms a known result, see for instance eq. (4.15) from ref. [53].

- Let $f_{p, q}(z)=z^{p}(1-z)^{-q}$. It will be instructive to spend some time on the computation of the alpha space density $\widehat{f}_{p, q}(\alpha)$. As a first step, we rewrite $\Psi_{\alpha}(z)$ using the MellinBarnes representation (2.52). This means that we can write

$$
\begin{aligned}
\widehat{f}_{p, q}(\alpha) & =\frac{1}{\Gamma\left(\frac{1}{2} \pm \alpha\right)} \int[d s] \frac{\Gamma(-s) \Gamma\left(\frac{1}{2} \pm \alpha+s\right)}{\Gamma(1+s)} \int_{0}^{1} \frac{d z}{z^{2}}\left(\frac{1-z}{z}\right)^{s} \frac{z^{p}}{(1-z)^{q}} \\
& =\frac{1}{\Gamma\left(\frac{1}{2} \pm \alpha\right) \Gamma(p-q)} \int[d s] \frac{\Gamma(-s) \Gamma\left(\frac{1}{2} \pm \alpha+s\right)}{\Gamma(1+s)} \Gamma(1-q+s) \Gamma(p-1-s)
\end{aligned}
$$

where in the first line we have interchanged the $z$ and $s$ integrals. What remains is a standard Mellin-Barnes integral, which evaluates to

$$
\begin{aligned}
\widehat{f}_{p, q}(\alpha)= & \frac{\Gamma(p-1) \Gamma(1-q)}{\Gamma(p-q)}{ }_{3} F_{2}\left(\begin{array}{c}
\frac{1}{2}+\alpha, \frac{1}{2}-\alpha, 1-q \\
1,2-p
\end{array} 1\right) \\
& +\frac{\Gamma(1-p) \Gamma\left(p-\frac{1}{2} \pm \alpha\right)}{\Gamma\left(\frac{1}{2} \pm \alpha\right) \Gamma(p)}{ }_{3} F_{2}\left(\begin{array}{c}
p-\frac{1}{2}+\alpha, p-\frac{1}{2}-\alpha, p-q \\
p, p
\end{array} ;\right) .
\end{aligned}
$$

which provides an analytic continuation to all $\alpha$, provided that $q>p-1 .^{10}$ Notice that the first term above is analytic in $\alpha$, hence it does not contain any poles in $\alpha$. However, it does influence the behaviour of $\widehat{f}_{p, q}(\alpha)$ at large $\alpha$. The second term contributes two series of poles, at $\pm \alpha=p-\frac{1}{2}+\mathbb{N}$. Closing the $\alpha$-contour to the right and computing residues, we arrive at the following conformal block decomposition:

$$
f_{p, q}(z)=\sum_{n=0}^{\infty} \frac{(p)_{n}^{2}}{n !(2 p-1+n)_{n}}{ }_{3} F_{2}\left(\begin{array}{c}
-n, 2 p-1+n, p-q \\
p, p
\end{array} ; 1\right) k_{p+n}(z) .
$$

This is a new result which would have been rather difficult to guess. For $p=q$, this reduces to eq. (4.14) from [53].

\footnotetext{
${ }^{10}$ Interestingly, the above expression can be analytically continued to other values of $p$ and $q$ using hypergeometric identities, in particular Thm. (2.4.4) and Corrollary (3.3.5) from [41]. We can for instance write

$$
\widehat{f}_{p, q}(\alpha)=\frac{\Gamma(1-q) \Gamma\left(p-\frac{1}{2} \pm \alpha\right)}{\Gamma(p) \Gamma(p-q)}{ }_{3} F_{2}\left(\begin{array}{cc}
\frac{1}{2}+\alpha, \frac{1}{2}-\alpha, q & ; 1 \\
p, 1 & ; 1
\end{array}\right)=\frac{\Gamma\left(p-\frac{1}{2} \pm \alpha\right)}{\Gamma^{2}(p)}{ }_{3} F_{2}\left(\begin{array}{c}
p-\frac{1}{2}+\alpha, p-\frac{1}{2}-\alpha, q \\
p, p
\end{array} ; 1\right) .
$$
}

The ${ }_{3} F_{2}(1)$ hypergeometrics in these expressions converge when $p>q$ resp. $q>1$. 


\subsection{Convergence and asymptotics}

In section 2.3 we discussed the convergence of the alpha space transform in a general setting. In the present section, we will specialize to CFT correlation functions, and more particularly, we will relate the large $\alpha$ behaviour of $\widehat{f}(\alpha)$ to the growth of $f(z)$ as $z \rightarrow 1$. Recall that at the extreme points $z=0$ and $z=1$ a crossing-symmetric four-point function in a unitary CFT behaves as

$$
\begin{aligned}
& z \rightarrow 0: F_{\phi \phi \phi \phi}(z) \rightarrow 1+\ldots \\
& z \rightarrow 1: F_{\phi \phi \phi \phi}(z) \rightarrow\left(\frac{z}{1-z}\right)^{-2 h_{\phi}}(1+\ldots)
\end{aligned}
$$

Clearly such a function is not square integrable with respect to the inner product (2.7). As we will now proceed to explain, an alpha space transform can nevertheless be defined also for such functions. We will show that divergences near the two endpoints $z=0$ and $z=1$ translate very differently into alpha space and bear resemblance to the usual IR and UV divergences in Fourier space.

Let us first focus on $z \rightarrow 0$, which is the OPE limit, and suppose we try to transform a function $f(z)$ behaving like $z^{p}(1+\ldots)$ for small $z$ to alpha space. For our inner product square integrability is lost as we dial $p$ to a value less than or equal to $1 / 2$. In alpha space this is reflected by a pair of poles crossing the real axis, as follows from the correspondence between conformal blocks of dimension $h$ and poles at $\alpha= \pm(h-1 / 2)$. This forces the integration contour in the inverse alpha transform off the imaginary axis, since the correct position-space expression is recovered only if it wraps around the poles as indicated in figure 2. This is however the only modification necessary, and we conclude that $z \rightarrow 0$ singularities of power-law form can be entirely dealt with by augmenting the inverse alpha space transform (2.17) with a contour prescription around the poles. This prescription works without issues for any $0<p<1 / 2$; the special cases $p=0$ and $p=1 / 2$ were discussed above in section 2.4.

Now let us consider the limit $z \rightarrow 1$. For simplicity we will restrict ourselves to the (physically relevant) case of functions $f(z)$ analytic in $0<z<1$. First of all, since $\Psi_{\alpha}(1)=1$ we find that

$$
f(1)=\int[d \alpha] \frac{\widehat{f}(\alpha)}{N(\alpha)},
$$

and similarly it follows from $D \cdot \Psi_{\alpha}(z)=\left(\alpha^{2}-1 / 4\right) \Psi_{\alpha}(z)$ that

$$
D^{n} \cdot f(1)=\int[d \alpha]\left(\alpha^{2}-1 / 4\right)^{n} \frac{\widehat{f}(\alpha)}{N(\alpha)},
$$

which holds as long as the $D^{n} \cdot f(z)$ remains square integrable. Supposing $f(z)$ behaves as a power law near $z=1$, we see from

$$
D \cdot\left((1-z)^{\rho}(1+\ldots)\right)=\rho^{2}(1-z)^{\rho-1}(1+\ldots),
$$

that acting with the Casimir operator $D$ worsens the behavior near $z=1$. For generic positive $\rho$ there exists an $n$ such that $D^{n} \cdot f(1)$ ceases to be well-defined, and therefore the 
integral in (2.62) should somehow suffer the same fate. Since we only modify the integrand with a polynomial factor, this can only happen if the integral stops converging. We conclude that the large alpha behavior reflects the 'short-distance' behavior of $f(z)$ as $z \rightarrow 1$. $^{11}$

The above discussion also offers a way to make sense of power-law divergent densities in alpha space: we just divide $\widehat{f}(\alpha)$ by sufficiently powers of $\alpha^{2}-1 / 4$, perform the nowconvergent integral over $\alpha$, and act just as many times with $D$ on the resulting positionspace expression. This is in fact entirely analogous to the usual trick in Fourier space, where we habitually make sense of UV-divergent expressions like $p^{2 \alpha}$ with $\alpha>0$ by replacing powers of $p^{2}$ with a Laplacian operator,

$$
\int d x e^{i p x} p^{2 \alpha}(1+\ldots) \rightarrow(-\square)^{n}\left(\int d x e^{i p x} p^{2 \alpha-2 n}(1+\ldots)\right),
$$

with $n$ chosen such that the integral becomes convergent at large $p$.

The relation between large $\alpha$ and $z$ close to 1 can be made more quantitative. Firstly, if a function $f(z)$ is infinitely differentiable at $z=1$, then the preceding logic demonstrates that $\widehat{f}(\alpha) / N(\alpha)$ must fall off faster than any power for large imaginary alpha. This is exemplified by the alpha space transform of $z^{\rho}$ given above, which falls off exponentially fast. Secondly, for the generic power-law behavior we find that if

$$
f(z)=(1-z)^{-\rho}(1+O(1-z)) \quad \text { then } \quad \widehat{f}(\alpha)=\left(-\alpha^{2}\right)^{\rho-1} \frac{\Gamma(1-\rho)}{\Gamma(\rho)}\left(1+O\left(\alpha^{-2}\right)\right),
$$

which can be found by subtracting the leading power using the alpha space transform of a known function. For example, for small enough $\rho$ one can use

$$
\begin{aligned}
\int \frac{d^{2} z}{z^{2}}\left[z^{\rho}(1-z)^{-\rho}-z^{\rho}\right] \Psi_{\alpha}(z) & =\frac{\Gamma\left(\rho-\frac{1}{2} \pm \alpha\right)}{\Gamma^{2}(\rho)}\left[\frac{\Gamma(1-\rho) \Gamma(\rho)}{\Gamma\left(\frac{1}{2} \pm \alpha\right)}-1\right] \\
& =\left(-\alpha^{2}\right)^{\rho-1} \frac{\Gamma(1-\rho)}{\Gamma(\rho)}\left(1+O\left(\alpha^{-2}\right)\right)
\end{aligned}
$$

which can be computed as a limit from the above examples.

\subsubsection{Application: OPE convergence}

We can use the preceding result to discuss the asymptotic behavior of OPE coefficients in one-dimensional CFTs, i.e., to provide a one-dimensional analogue of the results of $[10,66]$. Such a result has been discussed previously in the context of the light-cone limit for higherdimensional CFTs $[12,13]$. Here we offer an explanation based on the assumption of suitably nice asymptotic behavior in alpha space.

Consider once more a unitary CFT correlation function $F_{\phi \phi \phi \phi}(z)$ with a corresponding alpha space expression $F(\alpha)$ which is meromorphic with simple poles. Our preceding discussion leads us to conclude that $F(\alpha) \sim\left(-\alpha^{2}\right)^{2 h_{\phi}-1}$ for large imaginary $\alpha$, since

\footnotetext{
${ }^{11}$ We can also offer a physical explanation. For fixed alpha the $\Psi_{a}(z)$ oscillate very slowly near $z=1$ and to probe this region we need to consider very short 'wavelengths', corresponding to very large values of the 'momentum' $\alpha$.
} 
$F_{\phi \phi \phi \phi}(z) \sim(1-z)^{-2 h_{\phi}}$ as $z \rightarrow 1$. We will assume that this asymptotic behavior holds for all non-real $\alpha$ and so the 'subtracted' function

$$
F^{(s)}(\alpha):=\left(\alpha^{2}\right)^{-2 h_{\phi}+1-\epsilon} F(\alpha)
$$

vanishes asymptotically away from the real axis for any $\epsilon>0$. This means we can write a dispersion relation for it: we write

$$
F^{(s)}(\alpha)=\oint[d \beta] \frac{F^{(s)}(\beta)}{\alpha-\beta}
$$

and push the contour away from the point $\alpha$. With the arcs of the contour at infinity vanishing, we find contributions only from the cuts created by the power-law prefactor and the real axis where $F(\alpha)$ has poles. The contributions from the cuts can be made manifestly finite by aligning them along the imaginary axis and keeping the contour some distance away from $\alpha=0$. It follows that the contribution from the poles, which after picking up the residues can be written as

$$
\sum_{n}\left(\alpha_{n}^{2}\right)^{-2 h_{\phi}+1-\epsilon} R_{n}\left(\frac{1}{\alpha-\alpha_{n}}+(\alpha \leftrightarrow-\alpha)\right)
$$

is necessarily finite as well. In a distributional sense, then, we expect the residue series to behave as

$$
\sum_{n} \delta\left(h-h_{n}\right) R_{n} \sim c\left(h_{\phi}\right) h^{4 h_{\phi}-2}
$$

By working out the example given previously we also find the prefactor:

$$
c\left(h_{\phi}\right)=\frac{1}{\Gamma^{2}\left(2 h_{\phi}\right)} .
$$

We observe that the prefactor vanishes when $2 h_{\phi}$ is a negative integer which is precisely when the $z=1$ singularity in $F_{\phi \phi \phi \phi}(z)$ also disappears.

Finally we can use equation (2.46) and to relate this result to the asymptotic behavior of the squared primary OPE coefficients themselves as

$$
\lambda_{\phi \phi \mathcal{O}_{h}}(h)^{2} \sim \frac{4^{1-h} \sqrt{\pi}}{\Gamma^{2}\left(2 h_{\phi}\right)} h^{4 h_{\phi}-3 / 2} .
$$

agreeing with the lightcone bootstrap result, see e.g. [28]. ${ }^{12}$ It is interesting to see that the leading exponential falloff arises from the prefactor $Q(1 / 2-h)$, and the falloff speed is independent of the external dimension.

\footnotetext{
${ }^{12}$ Strictly speaking there is a factor 2 mismatch between (2.72) and formula (3.8) in [28], due to the fact that in the $d$-dimensional lightcone results only even spins are allowed to contribute.
} 


\subsection{Alpha space for different external dimensions}

So far we considered the case of a four-point function of identical operators. However, the Sturm-Liouville theory for the $\mathrm{SL}(2, \mathbb{R})$ Casimir operator applies just as well to four-point functions of different operators. In this section, we will briefly discuss this generalization.

Concretely, we have in mind a four-point function of primaries $\phi_{i}$ of dimension $h_{i}$, $i=1, \ldots, 4$. Conformal symmetry restricts this correlator to have the following form:

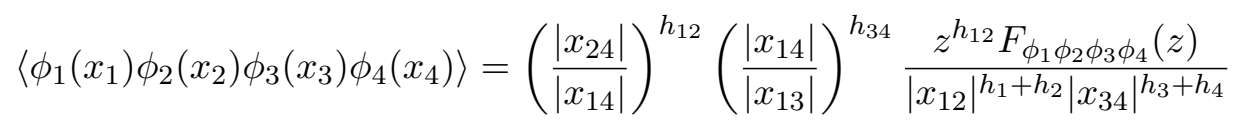

for some function $F_{\phi_{1} \phi_{2} \phi_{3} \phi_{4}}(z)$, using the shorthand $h_{i j} \equiv h_{i}-h_{j}$. The stripped correlator admits a conformal block decomposition of the following form:

$$
F_{\phi_{1} \phi_{2} \phi_{3} \phi_{4}}(z)=\sum_{\mathcal{O}} \lambda_{\phi_{1} \phi_{2} \mathcal{O}} \lambda_{\phi_{3} \phi_{4} \mathcal{O}} k_{h_{\mathcal{O}}}^{s}(z)
$$

involving the mixed $\mathrm{SL}(2, \mathbb{R})$ conformal blocks

$$
k_{h}^{s}(z)=z_{2}^{h+a} F_{1}(h+a, h+b ; 2 h ; z), \quad a=-h_{12}, b=h_{34} .
$$

The sum in eq. (2.74) now runs over all operators that appear in both the $\phi_{1} \times \phi_{2}$ and $\phi_{3} \times \phi_{4}$ OPEs; the label ' $s$ ' refers to this $s$-channel.

The blocks $k_{h}^{s}(z)$ are eigenfunctions of a mixed Casimir differential operator $D_{a, b}$ :

$$
D_{a, b} \cdot f(z)=w_{s}(z)^{-1} \frac{d}{d z}\left[w_{s}(z)(1-z) z^{2} f^{\prime}(z)\right]+a(a+1) f(z), \quad w_{s}(z)=\frac{(1-z)^{a+b}}{z^{2+2 a}}
$$

which means that $D_{a, b}$ is self-adjoint with respect to the inner product

$$
\langle f, g\rangle_{s}=\int_{0}^{1} d z w_{s}(z) \overline{f(z)} g(z) .
$$

Analyzing the relevant Sturm-Liouville problem leads to the following basis of eigenfunctions: ${ }^{13}$

$$
\Psi_{\alpha}^{s}(z)={ }_{2} F_{1}\left(\begin{array}{c}
\frac{1}{2}+a+\alpha, \frac{1}{2}+a-\alpha \\
1+a+b
\end{array} ; \frac{z-1}{z}\right)=\vartheta_{\alpha}^{(a+b, a-b)}\left(\frac{1-z}{z}\right) .
$$

In the second equality, we have rewritten $\Psi_{\alpha}^{s}(z)$ as a Jacobi function, to make contact with the integral transform introduced previously.

To connect the eigenfunctions $\Psi_{\alpha}^{s}(z)$ to the conformal blocks, we compute

$$
\Psi_{\alpha}^{s}(z)=\frac{1}{2}\left[Q_{s}(\alpha) k_{\frac{1}{2}+\alpha}^{s}(z)+(\alpha \rightarrow-\alpha)\right], \quad Q_{s}(\alpha)=\frac{2 \Gamma(-2 \alpha) \Gamma(1+a+b)}{\Gamma\left(\frac{1}{2}+a-\alpha\right) \Gamma\left(\frac{1}{2}+b-\alpha\right)} .
$$

\footnotetext{
${ }^{13}$ The PDE $D_{a, b} f(z)=\left(\alpha^{2}-1 / 4\right) f(z)$ has a second solution, namely

$$
\frac{z^{2 a}}{(1-z)^{a+b}}{ }_{2} F_{1}\left(\begin{array}{c}
\frac{1}{2}-a+\alpha, \frac{1}{2}-a-\alpha \\
1-a-b
\end{array} ; \frac{z-1}{z}\right) .
$$
}

This second solution ceases to be regular at $z=1$ when $a+b>0$. 
As in the case of equal external dimensions, we can decompose any function $f(z)$ - normalizable with respect to $(2.77)$ - in terms of the functions $\Psi_{\alpha}^{s}(z)$, to wit:

$$
f(z)=\int \frac{[d \alpha]}{N_{s}(\alpha)} \widehat{f}(\alpha) \Psi_{\alpha}^{s}(z) \quad \Leftrightarrow \quad \widehat{f}(\alpha)=\int_{0}^{1} d z w_{s}(z) f(z) \Psi_{\alpha}^{s}(z)
$$

where

$$
N_{s}(\alpha)=\frac{2 \Gamma( \pm 2 \alpha) \Gamma^{2}(1+a+b)}{\Gamma\left(\frac{1}{2}+a \pm \alpha\right) \Gamma\left(\frac{1}{2}+b \pm \alpha\right)}=\frac{\left|Q_{s}(\alpha)\right|^{2}}{2} .
$$

Some care must be taken when considering the $\alpha$ contour in eq. (2.80): when either $a, b \leq$ $-\frac{1}{2}$, the contour must be deformed in the Mellin-Barnes sense because of poles in the factor $1 / N_{s}(\alpha)$.

Cross channel. Applying crossing symmetry to mixed four-point functions leads to a relation between two different four-point functions. In the case of the correlator $\left\langle\phi_{1} \phi_{2} \phi_{3} \phi_{4}\right\rangle$, the bootstrap equation of interest is

$$
F_{\phi_{1} \phi_{2} \phi_{3} \phi_{4}}(z)=\left(\frac{z}{1-z}\right)^{2 h_{2}} F_{\phi_{3} \phi_{2} \phi_{1} \phi_{4}}(1-z)
$$

where $F_{\phi_{3} \phi_{2} \phi_{1} \phi_{4}}(z)$ is defined as in (2.73) but with $\phi_{1} \leftrightarrow \phi_{3}$ and $h_{1} \leftrightarrow h_{3}$ exchanged. Such mixed crossing equations have been used intensively in computing scaling dimensions and OPE coefficients for the $3 d$ Ising and $O(N)$ models [17, 24].

Like before, the correlator $F_{\phi_{3} \phi_{2} \phi_{1} \phi_{4}}(z)$ appearing in the r.h.s. of (2.82) admits a decomposition in conformal blocks and in plane-wave normalizable eigenfunctions of the conformal Casimir. However, care must be taken to use conformal blocks with dimensions $h_{1} \leftrightarrow h_{3}$ exchanged, and likewise for the eigenfunctions $\Psi_{\alpha}^{s}(z)$. To be completely explicit, this new conformal block decomposition reads:

$$
F_{\phi_{3} \phi_{2} \phi_{1} \phi_{4}}(z)=\sum_{\mathcal{O}} \lambda_{\phi_{2} \phi_{3} \mathcal{O}} \lambda_{\phi_{1} \phi_{4} \mathcal{O}} k_{h_{\mathcal{O}}}^{t}(z)
$$

with

$$
k_{h}^{t}(z)=z_{2}^{h+a^{\prime}} F_{1}\left(h+a^{\prime}, h+b^{\prime} ; 2 h ; z\right), \quad a^{\prime}=h_{23}, b^{\prime}=h_{14} .
$$

Here and in what follows we use the ' $t$ ' label for blocks and eigenfunctions in the $\phi_{2} \times \phi_{3} \rightarrow$ $\phi_{1} \times \phi_{4}$ channel. The appropriate eigenfunctions in the $t$-channel are

$$
\begin{aligned}
\left.\Psi_{\alpha}^{t}(z) \equiv \Psi_{\alpha}^{s}(z)\right|_{h_{1} \leftrightarrow h_{3}} & ={ }_{2} F_{1}\left(\begin{array}{c}
\frac{1}{2}+a^{\prime}+\alpha, \frac{1}{2}+a^{\prime}-\alpha \\
1+a^{\prime}+b^{\prime}
\end{array} ; \frac{z-1}{z}\right) \\
& =\vartheta_{\alpha}^{\left(a^{\prime}+b^{\prime}, a^{\prime}-b^{\prime}\right)}\left(\frac{1-z}{z}\right)
\end{aligned}
$$

which satisfy

$$
\Psi_{\alpha}^{t}(z)=\frac{1}{2}\left[Q_{t}(\alpha) k_{\frac{1}{2}+\alpha}^{t}(z)+(\alpha \rightarrow-\alpha)\right], \quad Q_{t}(\alpha)=\frac{2 \Gamma(-2 \alpha) \Gamma\left(1+a^{\prime}+b^{\prime}\right)}{\Gamma\left(\frac{1}{2}+a^{\prime}-\alpha\right) \Gamma\left(\frac{1}{2}+b^{\prime}-\alpha\right)}
$$


Finally, the decomposition of a function $f(z)$ in terms of the functions $\Psi_{\alpha}^{t}$ reads

$$
f(z)=\int \frac{[d \alpha]}{N_{t}(\alpha)} \widehat{f}(\alpha) \Psi_{\alpha}^{t}(z) \Leftrightarrow \widehat{f}(\alpha)=\int_{0}^{1} d z w_{t}(z) f(z) \Psi_{\alpha}^{t}(z)
$$

where

$$
w_{t}(z)=\frac{(1-z)^{a^{\prime}+b^{\prime}}}{z^{2+2 a^{\prime}}} \quad \text { and } \quad N_{t}(\alpha)=\frac{\left|Q_{t}(\alpha)\right|^{2}}{2} .
$$

\section{Crossing kernel}

So far, we have used Sturm-Liouville theory as a tool to represent conformal correlators as integrals over a set of basis functions $\Psi_{\alpha}$. In this section, we will use these integral representations to analyze crossing symmetry. In particular, we will compute the $d=1$ crossing kernel and exhibit its properties.

\subsection{General case}

Let us start by considering a mixed four-point function $\left\langle\phi_{1} \phi_{2} \phi_{3} \phi_{4}\right\rangle$. For such a correlator, we can write down two inequivalent integral representations:

$$
\begin{aligned}
\left\langle\phi_{1} \phi_{2} \phi_{3} \phi_{4}\right\rangle & \sim F_{\phi_{1} \phi_{2} \phi_{3} \phi_{4}}(z)=\int \frac{[d \alpha]}{N_{s}(\alpha)} F_{s}(\alpha) \Psi_{\alpha}^{s}(z), \\
\left\langle\phi_{3} \phi_{2} \phi_{1} \phi_{4}\right\rangle & \sim F_{\phi_{3} \phi_{2} \phi_{1} \phi_{4}}(z)=\int \frac{[d \alpha]}{N_{t}(\alpha)} F_{t}(\alpha) \Psi_{\alpha}^{t}(z) .
\end{aligned}
$$

The $\sim$ above denotes that we have omitted various unimportant scaling factors. The spectral density $F_{s}(\alpha)$ encodes information about the CB decomposition in the $s$-channel $\phi_{1} \times \phi_{2} \rightarrow \phi_{3} \times \phi_{4}$, whereas $F_{t}(\alpha)$ describes the $t$-channel $\phi_{1} \times \phi_{4} \rightarrow \phi_{2} \times \phi_{3}$.

The two alpha space densities $F_{s, t}(\alpha)$ are related - at least implicitly - via the crossing equation (2.82). Plugging eq. (3.1) into that equation, we find that

$$
\int \frac{[d \alpha]}{N_{s}(\alpha)} F_{s}(\alpha) \Psi_{\alpha}^{s}(z)=\left(\frac{z}{1-z}\right)^{2 h_{2}} \int \frac{[d \beta]}{N_{t}(\beta)} F_{t}(\beta) \Psi_{\beta}^{t}(1-z) .
$$

In order to find make the constraints on $F_{s, t}(\alpha)$ manifest, we can manipulate this alpha space bootstrap equation in various ways. For instance, it is possible to express $t$-channel eigenfunctions in terms of the $s$-channel ones:

$$
\left(\frac{z}{1-z}\right)^{2 h_{2}} \Psi_{\beta}^{t}(1-z)=\int \frac{[d \alpha]}{N_{s}(\alpha)} K\left(\alpha, \beta \mid h_{1}, h_{2}, h_{3}, h_{4}\right) \Psi_{\alpha}^{s}(z) .
$$

The distribution $K\left(\alpha, \beta \mid h_{1}, h_{2}, h_{3}, h_{4}\right)$ introduced here relates eigenfunctions in the $s$ - and t-channels, and we will refer to it as a crossing kernel. A schematic interpretation of eq. (3.3) is given in figure 3 .

Using (3.3), we can recast the crossing equation (3.2) as

$$
\int \frac{[d \alpha]}{N_{s}(\alpha)}\left[F_{s}(\alpha)-\left(\mathrm{K} \cdot F_{t}\right)(\alpha)\right] \Psi_{\alpha}^{s}(z)=0
$$



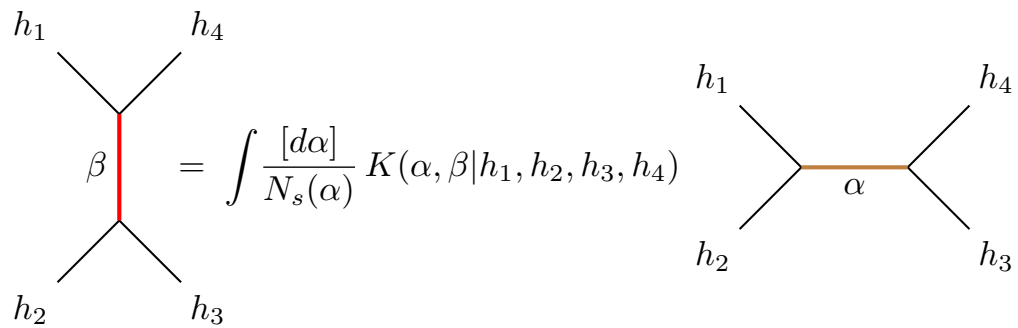

Figure 3. Graphical representation of the crossing kernel $K\left(\alpha, \beta \mid h_{1}, h_{2}, h_{3}, h_{4}\right)$.

where we have introduced an integral operator $\mathrm{K}$ which depends on the $h_{i}$ :

$$
(\mathrm{K} \cdot f)(\alpha):=\int \frac{[d \beta]}{N_{t}(\beta)} K\left(\alpha, \beta \mid h_{1}, h_{2}, h_{3}, h_{4}\right) f(\beta) .
$$

Recalling that the $\Psi_{\alpha}^{s}(z)$ form a complete basis in $z$-space, eq. (3.4) can only be satisfied if

$$
F_{s}(\alpha)=\left(\mathrm{K} \cdot F_{t}\right)(\alpha) \text {. }
$$

The point of this identity is that it directly relates the two densities $F_{s, t}(\alpha)$; once we compute the kernel $K\left(\alpha, \beta \mid h_{i}\right)$, eq. (3.6) will be completely explicit.

In the previous computation, we made an arbitrary choice by expressing $\Psi_{\beta}^{t}(1-z)$ in terms of the $s$-channel functions $\Psi_{\alpha}^{s}(z)$. It will be useful to go in the opposite direction as well, by writing

$$
\left(\frac{z}{1-z}\right)^{2 h_{2}} \Psi_{\beta}^{s}(1-z)=\int \frac{[d \alpha]}{N_{s}(\alpha)} \widetilde{K}\left(\alpha, \beta \mid h_{1}, h_{2}, h_{3}, h_{4}\right) \Psi_{\alpha}^{t}(z)
$$

which involves a second crossing kernel $\widetilde{K}\left(\alpha, \beta \mid h_{1}, \ldots, h_{4}\right)$. Using the same logic as before, we arrive at an alternate alpha space crossing equation:

$$
F_{t}(\alpha)=\left(\widetilde{\mathrm{K}} \cdot F_{s}\right)(\alpha)
$$

where

$$
(\widetilde{\mathrm{K}} \cdot f)(\alpha):=\int \frac{[d \beta]}{N_{s}(\beta)} \widetilde{K}\left(\alpha, \beta \mid h_{1}, h_{2}, h_{3}, h_{4}\right) f(\beta) .
$$

Bringing everything together, we have recast crossing symmetry as a system of integral equations in alpha space:

$$
F_{s}(\alpha)=\left(\mathrm{K} \cdot F_{t}\right)(\alpha), \quad F_{t}(\alpha)=\left(\widetilde{\mathrm{K}} \cdot F_{s}\right)(\alpha) .
$$

\subsection{Identical operators}

Let us briefly consider the case of the four-point function $\langle\phi \phi \phi \phi\rangle$ of four identical primaries. In that case, there is only one spectral density $F(\alpha)$ of interest, namely

$$
\langle\phi \phi \phi \phi\rangle \sim F_{\phi \phi \phi \phi}(z)=\int \frac{[d \alpha]}{N(\alpha)} F(\alpha) \Psi_{\alpha}(z) .
$$


Rather than a system of coupled integral equations, one now finds an eigenvalue equation for the density $F(\alpha)$ :

$$
F(\alpha)=\left(\mathrm{K}_{0} \cdot F\right)(\alpha)
$$

where the integral operator $\mathrm{K}_{0}$ is defined as

$$
\left(\mathrm{K}_{0} \cdot f\right)(\alpha):=\int \frac{[d \beta]}{N(\beta)} K_{0}\left(\alpha, \beta \mid h_{\phi}\right) f(\beta), \quad K_{0}\left(\alpha, \beta \mid h_{\phi}\right):=K\left(\alpha, \beta \mid h_{\phi}, h_{\phi}, h_{\phi}, h_{\phi}\right) .
$$

\subsection{Functional properties of the crossing kernels}

In what follows, we will compute the crossing kernels $K\left(\alpha, \beta \mid h_{i}\right), \widetilde{K}\left(\alpha, \beta \mid h_{i}\right)$ and $K_{0}\left(\alpha, \beta \mid h_{i}\right)$. Since this computation is somewhat technical, we will first derive several properties of these kernels.

Evidently, all of the kernels are even in their arguments $\alpha$ and $\beta$. Less trivially, we see that the kernels $K$ and $\widetilde{K}$ are identical after exchanging the external dimensions $h_{1}$ and $h_{3}$ :

$$
\widetilde{K}\left(\alpha, \beta \mid h_{1}, h_{2}, h_{3}, h_{4}\right)=K\left(\alpha, \beta \mid h_{3}, h_{2}, h_{1}, h_{4}\right)
$$

as follows from eqs. (3.3), (3.7).

Next, from the structure of eq. (3.10), we can surmise that

$$
\mathrm{K} \cdot \widetilde{\mathrm{K}}=\widetilde{\mathrm{K}} \cdot \mathrm{K}=\mathrm{id} .
$$

We have derived this with input from the bootstrap, but later we will rederive eq. (3.15) formally. For the case of identical operators, eq. (3.15) becomes

$$
\mathrm{K}_{0}^{2}=\mathrm{id}
$$

Notice that eqs. (3.15) and (3.16) only hold when restricted to some space of even functions, as the images of the integral operators $\mathrm{K}, \widetilde{\mathrm{K}}$ and $\mathrm{K}_{0}$ are even by construction.

Both identities (3.15) and (3.16) are statements about integral operators. By acting with these operators on test functions - say, having compact support - we can turn them into orthogonality/completeness relations for the crossing kernels themselves. To make this concrete, let's define the distributions

$$
\begin{aligned}
\mathscr{D}_{s}\left(\alpha, \beta \mid h_{1}, h_{2}, h_{3}, h_{4}\right) & :=N_{s}(\alpha)^{-1} \int \frac{[d y]}{N_{t}(y)} K\left(\alpha, y \mid h_{i}\right) \widetilde{K}\left(y, \beta \mid h_{i}\right), \\
\mathscr{D}_{t}\left(\alpha, \beta \mid h_{1}, h_{2}, h_{3}, h_{4}\right) & :=N_{t}(\alpha)^{-1} \int \frac{[d y]}{N_{s}(y)} \widetilde{K}\left(\alpha, y \mid h_{i}\right) K\left(y, \beta \mid h_{i}\right) \\
& =\mathscr{D}_{s}\left(\alpha, \beta \mid h_{3}, h_{2}, h_{1}, h_{4}\right) .
\end{aligned}
$$

Our claim is that $\mathscr{D}_{s, t}\left(\alpha, \beta \mid h_{i}\right)$ behave as delta functions on the imaginary axis. Indeed, eq. (3.15) implies that

$$
\int[d \beta]\left\{\begin{array}{l}
\mathscr{D}_{s}\left(\alpha, \beta \mid h_{i}\right) \\
\mathscr{D}_{t}\left(\alpha, \beta \mid h_{i}\right)
\end{array}\right\} f(\beta)=\frac{f(\alpha)+f(-\alpha)}{2}
$$


where $f(\alpha)$ is arbitrary. This can be thought of as the "local" version of (3.15). In the case of identical operators, we simply have

$$
\int[d \beta] \mathscr{D}_{0}\left(\alpha, \beta \mid h_{\phi}\right) f(\beta)=\frac{f(\alpha)+f(-\alpha)}{2}
$$

where

$$
\mathscr{D}_{0}\left(\alpha, \beta \mid h_{\phi}\right)=N(\alpha)^{-1} \int \frac{[d y]}{N(y)} K_{0}\left(\alpha, y \mid h_{\phi}\right) K_{0}\left(y, \beta \mid h_{\phi}\right) .
$$

eqs. (3.19) and (3.20) can be obtained as a limiting case of (3.18). Interestingly, eqs. (3.18) and (3.19) imply that the distributions $\mathscr{D}_{s, t}\left(\alpha, \beta \mid h_{i}\right)$ and $\mathscr{D}_{0}\left(\alpha, \beta \mid h_{\phi}\right)$ are identical and independent of external dimensions $h_{i}$ resp. $h_{\phi}$. As with the Fourier transform, the above identities mean that well-behaved functions $f(\alpha)$ can be decomposed in terms of the "basis functions" $K, \widetilde{K}$ and $K_{0}$, with computable coefficients.

\subsection{Computation of the crossing kernel}

Let us now turn to the computation of the crossing kernel $K\left(\alpha, \beta \mid h_{i}\right)$. To do so, we can use the alpha space technology from section 2.1 to write down a position-space integral representation for $K$, namely

$$
K\left(\alpha, \beta \mid h_{1}, h_{2}, h_{3}, h_{4}\right)=\int_{0}^{1} d z w_{s}(z)\left(\frac{z}{1-z}\right)^{2 h_{2}} \Psi_{\alpha}^{s}(z) \Psi_{\beta}^{t}(1-z) .
$$

It will be convenient to employ standard Mellin representations for the functions $\Psi_{\alpha}^{s, t}(z)$ :

$$
\begin{aligned}
\Psi_{\alpha}^{s}(z) & =\frac{\Gamma(1+a+b)}{\Gamma\left(\frac{1}{2}+a \pm \alpha\right)} \int[d s] \frac{\Gamma(-s) \Gamma\left(\frac{1}{2}+a+s \pm \alpha\right)}{\Gamma(1+a+b+s)}\left(\frac{1-z}{z}\right)^{s}, \\
\Psi_{\beta}^{t}(1-z) & =\frac{\Gamma\left(1+a^{\prime}+b^{\prime}\right)}{\Gamma\left(\frac{1}{2}+a^{\prime} \pm \beta\right)} \int[d t] \frac{\Gamma(-t) \Gamma\left(\frac{1}{2}+a^{\prime}+t \pm \beta\right)}{\Gamma\left(1+a^{\prime}+b^{\prime}+t\right)}\left(\frac{z}{1-z}\right)^{t} .
\end{aligned}
$$

Plugging these into (3.21), one obtains an integral representation of the form

$$
K\left(\alpha, \beta \mid h_{i}\right)=\int_{0}^{1} d z \int[d s] \int[d t] \ldots
$$

Exchanging the order of the integrals, the $z$-integral yields a beta function, whereas the resulting $t$-intergral can be performed using the second Barnes lemma. What remains is the following Mellin representation: ${ }^{14}$

$$
\begin{aligned}
& K\left(\alpha, \beta \mid h_{1}, h_{2}, h_{3}, h_{4}\right)=\frac{\Gamma(1+a+b) \Gamma\left(1+a^{\prime}+b^{\prime}\right)}{\Gamma\left(\frac{1}{2}+a \pm \alpha\right) \Gamma\left(\frac{1}{2}+b^{\prime} \pm \beta\right)} \\
& \times \int[d s] \frac{\Gamma(-s) \Gamma\left(\frac{1}{2}+a+s \pm \alpha\right)}{\Gamma(1+a+b+s)} \frac{\Gamma\left(2 h_{1}-1-s\right) \Gamma\left(\frac{3}{2}-h_{1}-h_{4}+s \pm \beta\right)}{\Gamma\left(2-h_{1}+h_{2}-h_{3}-h_{4}+s\right)} .
\end{aligned}
$$

This integral can be performed by closing the contour and picking up poles on the right half plane, at $s=\mathbb{N}$ and $s=2 h_{1}-1+\mathbb{N}$. The result is a sum of two hypergeometric ${ }_{4} F_{3}(1)$

\footnotetext{
${ }^{14} \mathrm{~A}$ different-looking representation can be found by doing the $s$-integral first.
} 
functions, and it can be cast into a standard form by introducing the Wilson functions of ref. [67]: ${ }^{15}$

$$
\begin{aligned}
& \mathrm{W}_{\alpha}(\beta ; a, b, c, d)= \\
& =\frac{\Gamma(d-a)}{\Gamma(a+b) \Gamma(a+c) \Gamma(d \pm \beta) \Gamma(\tilde{d} \pm \alpha)}{ }_{4} F_{3}\left(\begin{array}{c}
a+\beta, a-\beta, \tilde{a}+\alpha, \tilde{a}-\alpha \\
a+b, a+c, 1+a-d
\end{array} ; 1\right)+(a \leftrightarrow d)
\end{aligned}
$$

writing $\tilde{a}=\frac{1}{2}(a+b+c-d)$ and $\tilde{d}=\frac{1}{2}(-a+b+c+d)$. It is useful to know that $\mathrm{W}_{\alpha}(\beta ; a, b, c, d)$ is even in its arguments $\alpha$ and $\beta$, and it depends symmetrically on its parameters $\{a, b, c, d\}$. A closed-form expression for the crossing kernel is then given by

$$
\begin{aligned}
K\left(\alpha, \beta \mid h_{1}, h_{2}, h_{3}, h_{4}\right)= & \Gamma\left(1-h_{12}+h_{34}\right) \Gamma\left(1+h_{14}+h_{23}\right) \\
& \times \Gamma\left(h_{1}+h_{2}-\frac{1}{2} \pm \alpha\right) \Gamma\left(\frac{3}{2}-h_{1}-h_{4} \pm \beta\right) \mathbf{W}_{\alpha}(\beta ; \mathscr{P})
\end{aligned}
$$

with parameters $\mathscr{P}=\mathscr{P}\left(h_{1}, h_{2}, h_{3}, h_{4}\right)$ specified by

$$
\mathscr{P}=\left\{\frac{1}{2}+h_{14}, \frac{1}{2}+h_{23}, h_{2}+h_{3}-\frac{1}{2}, \frac{3}{2}-h_{1}-h_{4}\right\} .
$$

The kernel $\widetilde{K}\left(\alpha, \beta \mid h_{1}, h_{2}, h_{3}, h_{4}\right)$ admits an expression similar to (3.26), the only difference being that $h_{1} \leftrightarrow h_{3}$ are swapped. For completeness, we print the formula for the identicaloperator kernel $K_{0}\left(\alpha, \beta \mid h_{\phi}\right)$ here as well:

$$
\begin{aligned}
K_{0}\left(\alpha, \beta \mid h_{\phi}\right) & =\Gamma\left(2 h_{\phi}-\frac{1}{2} \pm \alpha\right) \Gamma\left(\frac{3}{2}-2 h_{\phi} \pm \beta\right) \mathrm{W}_{\alpha}\left(\beta ; \mathscr{P}_{0}\right), \\
\mathscr{P}_{0} & =\left\{\frac{1}{2}, \frac{1}{2}, 2 h_{\phi}-\frac{1}{2}, \frac{3}{2}-2 h_{\phi}\right\} .
\end{aligned}
$$

\section{5 $\mathrm{K}$ and $\widetilde{\mathrm{K}}$ as intertwiners}

Having computed the crossing kernels $K$ and $\widetilde{K}$, let us now revisit the alpha space crossing equation (3.10). Informally, it encodes that $\mathrm{K}$ maps a " $t$-channel" alpha space density to an "s-channel" one, and vice versa for $\widetilde{K}$. In this section we will formalize this idea, making precise in which sense $\mathrm{K}$ and $\widetilde{\mathrm{K}}$ intertwine between two different Hilbert spaces.

First, let's introduce a Hilbert space $\mathcal{H}_{s}=\mathcal{H}_{s}\left(h_{1}, h_{2}, h_{3}, h_{4}\right)$ for $s$-channel functions, consisting of all functions $f(\alpha)$ that are even in $\alpha$ and $L^{2}$ with respect to the following inner product:

$$
\begin{aligned}
(f, g)_{s} & :=\int \frac{[d \alpha]}{\mathcal{M}_{s}\left(\alpha ; h_{1}, h_{2}, h_{3}, h_{4}\right)} \overline{f(\alpha)} g(\alpha), \\
\mathcal{M}_{s}\left(\alpha ; h_{1}, h_{2}, h_{3}, h_{4}\right) & =\frac{2 \Gamma^{2}\left(1-h_{12}+h_{34}\right) \Gamma( \pm 2 \alpha) \Gamma\left(h_{1}+h_{2}-\frac{1}{2} \pm \alpha\right)}{\Gamma\left(\frac{1}{2}-h_{12} \pm \alpha\right) \Gamma\left(\frac{1}{2}+h_{34} \pm \alpha\right) \Gamma\left(\frac{3}{2}-h_{3}-h_{4} \pm \alpha\right)} .
\end{aligned}
$$

We have introduced an $\alpha$-independent factor in the measure $\mathcal{M}_{s}\left(\alpha ; h_{i}\right)$ to simplify some formulas later on. The integration contour in (3.29) is to be understood in the MellinBarnes sense, which means that it may be deformed depending on the values of the $h_{i}$.

\footnotetext{
${ }^{15}$ Our conventions differ from those of [67] as follows: $\mathrm{W}_{\alpha}(\beta \mid a, b, c, d)=\phi_{i \alpha}(i \beta ; a, b, c, 1-d)$.
} 
Likewise, we introduce a $t$-channel Hilbert space $\mathcal{H}_{t}\left(h_{1}, h_{2}, h_{3}, h_{4}\right)$ of even functions that are square-integrable with respect to

$$
(f, g)_{t}:=\int \frac{[d \alpha]}{\mathcal{M}_{t}\left(\alpha ; h_{1}, h_{2}, h_{3}, h_{4}\right)} \overline{f(\alpha)} g(\alpha), \quad \mathcal{M}_{t}\left(\alpha ; h_{1}, h_{2}, h_{3}, h_{4}\right)=\mathcal{M}_{s}\left(\alpha ; h_{3}, h_{2}, h_{1}, h_{4}\right) .
$$

We now claim that the following holds:

Theorem 1.1: $\quad \mathrm{K}$ is a unitary map $\mathcal{H}_{t} \rightarrow \mathcal{H}_{s}$, and $\widetilde{\mathrm{K}}: \mathcal{H}_{s} \rightarrow \mathcal{H}_{t}$ is its inverse.

Unitarity here means that $\mathrm{K}$ and $\widetilde{K}$ preserve the inner products defined in eqs. (3.29) and (3.30), namely

$$
(f, g)_{t}=(\mathrm{K} \cdot f, \mathrm{~K} \cdot g)_{s} \text { and }(f, g)_{s}=(\widetilde{\mathrm{K}} \cdot f, \widetilde{\mathrm{K}} \cdot g)_{t} .
$$

The proof of this result follows from the properties of the Wilson transform, introduced in ref. [67]. This integral transform uses the Wilson functions $\mathrm{W}_{\alpha}(\beta ; a, b, c, d)$ as a basis. The above result can straightforwardly be deduced from Theorem 4.12 of ref. [67]. Consequently, we will not provide many details. However, it will be instructive to provide a sketch of a (constructive) proof. First, one establishes that $\mathcal{H}_{s}$ is spanned by the following functions:

$$
\xi_{n}^{s}\left(\alpha \mid h_{i}\right)=\Gamma\left(1-h_{12}+h_{34}\right) \Gamma\left(h_{1}+h_{2}-\frac{1}{2} \pm \alpha\right) \mathfrak{p}_{n}(\alpha ; \tilde{\mathscr{P}}), \quad n \in \mathbb{N} .
$$

The Wilson polynomials $\mathfrak{p}_{n}$ were defined in eq. (2.30), and the set of parameters $\tilde{\mathscr{P}}$ is given by

$$
\tilde{\mathscr{P}}\left(h_{1}, h_{2}, h_{3}, h_{4}\right)=\left\{\frac{1}{2}-h_{12}, \frac{1}{2}+h_{34}, h_{1}+h_{2}-\frac{1}{2}, \frac{3}{2}-h_{3}-h_{4}\right\}=\mathscr{P}\left(h_{3}, h_{2}, h_{1}, h_{4}\right) .
$$

Likewise, $\mathcal{H}_{t}$ is spanned by the functions

$$
\xi_{n}^{t}\left(\alpha \mid h_{i}\right)=\Gamma\left(1+h_{14}+h_{23}\right) \Gamma\left(h_{2}+h_{3}-\frac{1}{2} \pm \alpha\right) \mathfrak{p}_{n}(\alpha ; \mathscr{P}) .
$$

By linearity, it suffices to establish that $\mathrm{K}$ and $\widetilde{\mathrm{K}}$ act appropriately on these basis functions. To establish this, one proves first that

$$
\left(\xi_{m}^{s}, \xi_{n}^{s}\right)_{s}=\left(\xi_{m}^{t}, \xi_{n}^{t}\right)_{t} \propto \delta_{m n}
$$

as well as

$$
\left(\mathrm{K} \cdot \xi_{n}^{t}\right)(\alpha)=(-1)^{n} \xi_{n}^{s}(\alpha), \quad\left(\widetilde{\mathrm{K}} \cdot \xi_{n}^{s}\right)(\alpha)=(-1)^{n} \xi_{n}^{t}(\alpha) .
$$

eq. (3.35) is a property of the Wilson polynomials $\mathfrak{p}_{n}$ [41], and eq. (3.36) is a consequence of Theorem 6.7 of [67].

A similar result holds for the case of identical operators. There one defines a Hilbert space $\mathcal{H}_{0}=\mathcal{H}_{0}\left(h_{\phi}\right)$ of even functions that are finite with respect to

$$
(f, g)_{0}:=\int \frac{[d \alpha]}{\mathcal{M}_{0}\left(\alpha ; h_{\phi}\right)} \overline{f(\alpha)} g(\alpha), \quad \mathcal{M}_{0}\left(\alpha ; h_{\phi}\right)=\mathcal{M}_{s}\left(\alpha ; h_{\phi}, h_{\phi}, h_{\phi}, h_{\phi}\right) .
$$

Then the counterpart of the above theorem reads: 
Theorem 1.2: $\quad \mathrm{K}_{0}$ is a unitary map $\mathcal{H}_{0} \rightarrow \mathcal{H}_{0}$ obeying $\mathrm{K}_{0}^{2}=\mathrm{id}$.

Here unitarity means that

$$
(f, g)_{0}=\left(\mathrm{K}_{0} \cdot f, \mathrm{~K}_{0} \cdot g\right)_{0} .
$$

The proof goes along the same lines as the general case discussed before. A basis for $\mathcal{H}_{0}$ is now spanned by the functions

$$
\xi_{n}^{0}\left(\alpha \mid h_{\phi}\right)=\Gamma\left(2 h_{\phi}-\frac{1}{2} \pm \alpha\right) \mathfrak{p}_{n}\left(\alpha ; \mathscr{P}_{0}\right)
$$

where $\mathscr{P}_{0}$ was defined in (3.28). The operator $\mathrm{K}_{0}$ maps the $\xi_{n}^{0}$ to themselves, up to a sign $(-1)^{n}$ :

$$
\left(\mathrm{K}_{0} \cdot \xi_{n}^{0}\right)(\alpha)=(-1)^{n} \xi_{n}^{0}(\alpha) .
$$

Of course, the only permissible eigenvalues that could have appeared were \pm 1 , given that $\mathrm{K}_{0}^{2}=\mathrm{id}$.

\subsection{Analytic structure of the crossing kernel}

Since we have rephrased bootstrap equations as integral equations in alpha space, it will be instructive to analyze the analytic structure of the crossing kernel $K\left(\alpha, \beta \mid h_{1}, h_{2}, h_{3}, h_{4}\right)$. Let's first fix $\beta$ and investigate the properties of $K$ as a function of $\alpha$, using eq. (3.26). Since the Wilson functions $\mathrm{W}_{\alpha}(\beta ; a, b, c, d)$ are analytic in $\alpha$ and $\beta$, the only poles in $\alpha$ are due to the factor $\Gamma\left(h_{1}+h_{2}-\frac{1}{2} \pm \alpha\right)$. Consequently $K\left(\alpha, \beta \mid h_{i}\right)$ is a meromorphic function, with its only poles on the right half plane at $\alpha=h_{1}+h_{2}-\frac{1}{2}+\mathbb{N}$. The relevant residues are polynomials of degree $n$ in $\beta^{2}$, namely

$$
\begin{aligned}
\mathcal{R}_{n}\left(\beta ; h_{1}, h_{2}, h_{3}, h_{4}\right):= & -\left.\operatorname{Res} K\left(\alpha, \beta \mid h_{1}, h_{2}, h_{3}, h_{4}\right)\right|_{\alpha=h_{1}+h_{2}-1 / 2+n} \\
= & \frac{\Gamma\left(1-h_{12}+h_{34}\right)}{n !\left(1+h_{14}+h_{23}\right)_{n}} \frac{\Gamma\left(2 h_{1}+2 h_{2}-1+n\right)}{\Gamma\left(2 h_{2}+n\right) \Gamma\left(h_{1}+h_{2}+h_{34}+n\right)} \\
& \times \mathfrak{p}_{n}\left(\beta ; \frac{1}{2}+h_{14}, \frac{1}{2}+h_{23}, h_{1}+h_{4}-\frac{1}{2}, h_{2}+h_{3}-\frac{1}{2}\right) .
\end{aligned}
$$

Next, remark that for generic values of $\alpha, K\left(\alpha, \beta \mid h_{i}\right)$ is a rather complicated function of $\beta$. Upon closer inspection it appears that at certain values $\alpha_{*}$ the kernel $K\left(\alpha_{*}, \beta \mid h_{i}\right)$ becomes polynomial in $\beta$, up to a number of gamma functions. The relevant values $\alpha=\alpha_{*}$ are organized in three families:

$$
\alpha_{n}^{\mathrm{I}}=\frac{3}{2}-h_{3}-h_{4}+n, \quad \alpha_{n}^{\mathrm{II}}=\frac{1}{2}-h_{12}+n, \quad \alpha_{n}^{\mathrm{III}}=\frac{1}{2}+h_{34}+n, \quad n \in \mathbb{N} .
$$

For the first family, we find for instance

$$
K\left(\alpha_{n}^{\mathrm{I}}, \beta\right)=k_{n}^{\mathrm{I}} \frac{\Gamma\left(\frac{3}{2}-h_{1}-h_{4} \pm \beta\right)}{\Gamma\left(h_{2}+h_{3}-\frac{1}{2} \pm \beta\right)} \mathfrak{p}_{n}\left(\beta ; \frac{1}{2}+h_{14}, \frac{1}{2}+h_{23}, \frac{3}{2}-h_{1}-h_{4}, \frac{3}{2}-h_{2}-h_{3}\right)
$$


where $k_{n}^{\mathrm{I}}$ is a constant that does not depend on $\beta$. For the second and third families, we find

$$
\begin{gathered}
K\left(\alpha_{n}^{\mathrm{II}}, \beta\right)=k_{n}^{\mathrm{II}} \frac{\Gamma\left(\frac{3}{2}-h_{1}-h_{4} \pm \beta\right)}{\Gamma\left(\frac{1}{2}+h_{14} \pm \beta\right)} \mathfrak{p}_{n}\left(\beta ; \frac{1}{2}-h_{14}, \frac{1}{2}+h_{23}, h_{2}+h_{3}-\frac{1}{2}, \frac{3}{2}-h_{1}-h_{4}\right), \\
K\left(\alpha_{n}^{\mathrm{III}}, \beta\right)=k_{n}^{\mathrm{III}} \frac{\Gamma\left(\frac{3}{2}-h_{1}-h_{4} \pm \beta\right)}{\Gamma\left(\frac{1}{2}+h_{23} \pm \beta\right)} \mathfrak{p}_{n}\left(\beta ; \frac{1}{2}+h_{14}, \frac{1}{2}-h_{23}, h_{2}+h_{3}-\frac{1}{2}, \frac{3}{2}-h_{1}-h_{4}\right) .
\end{gathered}
$$

We can also consider the analytic structure of $K\left(\alpha, \beta \mid h_{i}\right)$ as a function of $\beta$ for fixed $\alpha$. This is a simple exercise, given the relation (3.14). We therefore refrain from printing explicit formulas.

\subsection{Symmetries of the crossing kernel}

The crossing kernel obeys various identities which we will exhibit here. Since none of these results are used in the rest of this paper, this section can be skipped on a first reading.

It will be convenient to strip off the gamma functions in eq. (3.26) and to relabel the external dimensions as $h_{i} \rightarrow \frac{1}{2}+\gamma_{i}$. What remains is a single Wilson function, namely

$$
\hat{K}\left(\alpha, \beta \mid \gamma_{1}, \gamma_{2}, \gamma_{3}, \gamma_{4}\right)=\mathbf{W}_{\alpha}\left(\beta \mid \frac{1}{2}+\gamma_{1}-\gamma_{4}, \frac{1}{2}+\gamma_{2}-\gamma_{3}, \frac{1}{2}-\gamma_{1}-\gamma_{4}, \frac{1}{2}+\gamma_{2}+\gamma_{3}\right) .
$$

First, we recall that $\mathrm{W}_{\alpha}(\beta ; a, b, c, d)$ depends symmetrically on its parameters $\{a, b, c, d\}$, which implies that $\hat{K}\left(\alpha, \beta \mid \gamma_{i}\right)$ obeys

$$
\begin{aligned}
\hat{K}\left(\alpha, \beta \mid \gamma_{1}, \gamma_{2}, \gamma_{3}, \gamma_{4}\right) & =\hat{K}\left(\alpha, \beta \mid-\gamma_{1}, \gamma_{2}, \gamma_{3}, \gamma_{4}\right)=\hat{K}\left(\alpha, \beta \mid \gamma_{1}, \gamma_{2},-\gamma_{3}, \gamma_{4}\right) \\
& =\hat{K}\left(\alpha, \beta \mid \gamma_{3},-\gamma_{4}, \gamma_{1},-\gamma_{2}\right) \\
& =\hat{K}\left(\alpha, \beta \mid-\gamma_{1}^{\natural},-\gamma_{2}^{\natural},-\gamma_{3}^{\natural},-\gamma_{4}^{\natural}\right), \quad \gamma_{i}^{\natural}=-\gamma_{i}+\frac{1}{2} \sum_{j=1}^{4} \gamma_{j} .
\end{aligned}
$$

A second type of symmetry can be found using the identity (see Lemma 5.3 of [68])

$$
\mathrm{W}_{\alpha}(\beta ; A+\omega, A-\omega, B+\rho, B-\rho)=\mathrm{W}_{\omega}(\rho ; A+\alpha, A-\alpha, B+\beta, B-\beta)
$$

which descends to

$$
\hat{K}\left(\alpha, \beta \mid \gamma_{1}, \gamma_{2}, \gamma_{3}, \gamma_{4}\right)=\hat{K}\left(\gamma_{1}, \gamma_{3} \mid \alpha, \gamma_{2}, \beta, \gamma_{4}\right) .
$$

A final relation follows from the "duality" property of the Wilson functions:

$$
\mathrm{W}_{\alpha}(\beta ; a, b, c, d)=\mathrm{W}_{\beta}(\alpha ; \tilde{a}, \tilde{b}, \tilde{c}, \tilde{d}), \quad\left[\begin{array}{c}
\tilde{a} \\
\tilde{b} \\
\tilde{c} \\
\tilde{d}
\end{array}\right]=\frac{1}{2}(a+b+c+d)-\left[\begin{array}{l}
d \\
c \\
b \\
a
\end{array}\right]
$$


which implies that

$$
\hat{K}\left(\alpha, \beta \mid \gamma_{1}, \gamma_{2}, \gamma_{3}, \gamma_{4}\right)=\hat{K}\left(\beta, \alpha \mid \gamma_{3}, \gamma_{2}, \gamma_{1}, \gamma_{4}\right)
$$

The reader may notice that the above symmetries are reminiscent of those corresponding to the $\mathrm{SU}(2) 6-j$ symbol [69-72]. In the $\mathrm{SU}(2)$ context, the transformations $\gamma_{1,3} \mapsto-\gamma_{1,3}$ are known as mirror symmetries and $\gamma_{i} \mapsto \gamma_{i}^{\natural}$ is a Regge transformation; eqs. (3.45b) (3.47) and (3.49) are related to transformations that exchange rows and columns of the $6-j$ symbol.

A subset of the above symmetries lifts to the full crossing kernel $K\left(\alpha, \beta \mid h_{i}\right)$ :

$$
\begin{aligned}
K\left(\alpha, \beta \mid h_{1}, h_{2}, h_{3}, h_{4}\right) & =K\left(\alpha, \beta \mid h_{3}^{\natural}, h_{4}^{\natural}, h_{1}^{\natural}, h_{2}^{\natural}\right), \quad h_{i}^{\natural}=-h_{i}+\frac{1}{2} \sum_{j=1}^{4} h_{j}, \\
& =K\left(\beta, \alpha \mid 1-h_{3}^{\natural}, 1-h_{2}^{\natural}, 1-h_{1}^{\natural}, 1-h_{4}^{\natural}\right), \\
& =K\left(\beta, \alpha \mid 1-h_{1}, 1-h_{4}, 1-h_{3}, 1-h_{2}\right) .
\end{aligned}
$$

Any two of these identities imply the third one. In conclusion, it appears that the automorphism group of the $K\left(\alpha, \beta \mid h_{i}\right)$ is isomorphic to the Klein four-group. In passing, we note that eq. (3.50) can also be derived by inspecting the integral representation (3.3).

Limit cases. For bootstrap applications, one is often interested in four-point functions where some of the operators are identical. In that case, the discussion of the symmetries of the crossing kernel simplifies drastically. For a mixed four-point function of the form $\langle\epsilon \sigma \sigma \epsilon\rangle$, there are two relevant crossing kernels:

$$
K_{\mathrm{m}, 1}\left(\alpha, \beta \mid h_{\sigma}, h_{\epsilon}\right):=K\left(\alpha, \beta \mid h_{\epsilon}, h_{\sigma}, h_{\sigma}, h_{\epsilon}\right), \quad K_{\mathrm{m}, 2}\left(\alpha, \beta \mid h_{\sigma}, h_{\epsilon}\right):=K\left(\alpha, \beta \mid h_{\sigma}, h_{\sigma}, h_{\epsilon}, h_{\epsilon}\right) .
$$

In this case, the content of eq. (3.50) reduces to

$$
K_{\mathrm{m}, 1}\left(\alpha, \beta \mid h_{\sigma}, h_{\epsilon}\right)=K_{\mathrm{m}, 2}\left(\beta, \alpha \mid 1-h_{\epsilon}, 1-h_{\sigma}\right) .
$$

Finally, when all external dimensions are identical, the relevant kernel is $K_{0}\left(\alpha, \beta \mid h_{\phi}\right)$, which obeys

$$
K_{0}\left(\alpha, \beta \mid h_{\phi}\right)=K_{0}\left(\beta, \alpha \mid 1-h_{\phi}\right)
$$

\section{Applications to the conformal bootstrap}

In section 3, we reformulated crossing symmetry in the form of integral equations in alpha space, making use of the crossing kernel $K\left(\alpha, \beta \mid h_{i}\right)$. For definiteness, let us consider the identical-operator alpha space equation eq. (3.12):

$$
F(\alpha)=\int \frac{[d \beta]}{N(\beta)} K_{0}\left(\alpha, \beta \mid h_{\phi}\right) F(\beta) .
$$

In the bootstrap context, we can ask whether eq. (4.1) (combined with unitarity) can be used to find useful constraints on $F(\alpha)$. In this section we will sketch some ideas in this direction, making use of the properties of the crossing kernel as discussed in section 3 . 


\section{1 (Dis)proving a false theorem}

We will start by outlining an simple idea for analyzing the alpha space crossing equation (4.1). One can think of the r.h.s. of (4.1) as a function of $\alpha$

$$
\alpha \mapsto \int \frac{[d \beta]}{N(\beta)} K_{0}\left(\alpha, \beta \mid h_{\phi}\right) F(\beta)
$$

and require that (4.2) has exactly the same analytic structure as $F(\alpha)$, appearing on the 1.h.s. of (4.1). Taken at face value, this should lead to constraints of the poles and residues of $F(\alpha)$, which correspond to CFT data.

The function (4.2) only depends on $\alpha$ through the crossing kernel $K_{0}\left(\alpha, \beta \mid h_{\phi}\right)$. Using the results of section 3.6, we see that the identical-operator kernel $K_{0}\left(\alpha, \beta \mid h_{\phi}\right)$ has poles at $\alpha_{n}=2 h_{\phi}-\frac{1}{2}+n, n \in \mathbb{N}$, with residues

$$
\mathscr{R}_{n}\left(\beta \mid h_{\phi}\right):=\mathcal{R}_{n}\left(\beta \mid h_{\phi}, h_{\phi}, h_{\phi}, h_{\phi}\right)=\frac{\Gamma\left(4 h_{\phi}-1+n\right)}{n !^{2} \Gamma^{2}\left(2 h_{\phi}+n\right)} \mathfrak{p}_{n}\left(\beta ; \frac{1}{2}, \frac{1}{2}, 2 h_{\phi}-\frac{1}{2}, 2 h_{\phi}-\frac{1}{2}\right) .
$$

Plugging this result into (4.1), we naively conclude that $F(\alpha)$ can only have poles at $\alpha=\alpha_{n}$, with their residues constrained as follows:

$$
-\left.\operatorname{Res} F(\alpha)\right|_{\alpha=\alpha_{n}} \stackrel{?}{=} \int \frac{[d \beta]}{N(\beta)} \mathscr{R}_{n}\left(\beta \mid h_{\phi}\right) F(\beta) .
$$

Obviously, this conclusion is wrong: it says that any solution to crossing consists of a single tower of exchanged operators with dimensions $2 h_{\phi}+\mathbb{N}$. Although solutions of this form exist (e.g. in mean field theory), any interacting CFT correlator furnishes a counterexample to (4.4). From a mathematical point of view, we have arrived at (4.4) using a doubtful manipulation:

$$
\operatorname{Res}\left[\int \frac{[d \beta]}{N(\beta)} K_{0}\left(\alpha, \beta \mid h_{\phi}\right) F(\beta)\right]_{\alpha=\alpha_{n}} \stackrel{?}{=} \int \frac{[d \beta]}{N(\beta)}\left[\operatorname{Res} K_{0}\left(\alpha, \beta \mid h_{\phi}\right)\right]_{\alpha=\alpha_{n}} F(\beta) \text {. }
$$

This fails to hold at general $\alpha$, as the function (4.2) is defined for real $\alpha$ only by analytic continuation. It would be interesting to see if this wrong argument can be refined to give useful bootstrap constraints, likely by deforming the contour in eq. (4.1), as discussed in section 2.4.

\subsection{Split kernel}

A second idea is to close the $\beta$ contour in eq. (4.1) to the right, picking up poles in $\beta$. Since the integrand appearing in the r.h.s. of (4.1) equals

$$
\frac{K_{0}\left(\alpha, \beta \mid h_{\phi}\right) F(\beta)}{N(\beta)}
$$

poles in $\beta$ can come from three different factors. As mentioned, the poles in $F(\beta)-$ and their residues - are unknown, but of physical interest. Next, $1 / N(\beta)$ has poles at 
$\beta=1 / 2+\mathbb{N}$, and $K_{0}\left(\alpha, \beta \mid h_{\phi}\right)$ has poles at $\beta=3 / 2-2 h_{\phi}+\mathbb{N} .{ }^{16}$ Closing the contour means that we have to keep track of all of these different poles.

We propose to modify eq. (4.1) in a straightforward way, bypassing this bookkeeping exercise. The key point is that both $N(\beta)$ and $F(\beta)$ are even in $\beta$; in the definition (3.3) of the crossing kernel, it is therefore possible to replace $\Psi_{\beta}^{t}(1-z)$ by $Q_{t}(\beta) k_{\frac{1}{2}+\beta}^{t}(1-z)$, where $Q_{t}$ and $k_{\alpha}^{t}(z)$ were defined in section 2.6. Concretely, we recast the crossing equation as

$$
\begin{aligned}
F(\alpha) & =\int[d \beta] K_{\text {split }}\left(\alpha, \beta \mid h_{\phi}, h_{\phi}, h_{\phi}, h_{\phi}\right) F(\beta), \\
K_{\text {split }}\left(\alpha, \beta \mid h_{1}, h_{2}, h_{3}, h_{4}\right) & :=\frac{Q_{t}(\beta)}{N_{t}(\beta)} \int_{0}^{1} d z w_{s}(z)\left(\frac{z}{1-z}\right)^{2 h_{2}} \Psi_{\alpha}^{s}(z) k_{\frac{1}{2}+\beta}^{t}(1-z) .
\end{aligned}
$$

We will from now on consider this "split" kernel $K_{\text {split }}\left(\alpha, \beta \mid h_{i}\right)$ with arbitrary external dimensions, although only the case $h_{1}=\ldots=h_{4} \equiv h_{\phi}$ is of interest in the analysis of eq. (4.1).

We claim that the split kernel $K_{\text {split }}$ does not have any poles on the right half plane $\Re(\beta)>0$. That is to say, by closing the contour of (4.7) to the right, we only pick up poles coming from $F(\beta)$, as desired.

The proof of this claim follows from a direct computation. The computation is very similar to the one from section 3.4. The only difference is that we use a Mellin-Barnes representation for the cross-channel block $k_{h}^{t}(1-z)$, namely

$$
\begin{aligned}
k_{\frac{1}{2}+\beta}^{t}(1-z)= & \frac{\Gamma(1+2 \beta)}{\Gamma\left(\frac{1}{2}+a^{\prime}+\beta\right) \Gamma\left(\frac{1}{2}-b^{\prime}+\beta\right)} \\
& \times \int[d t] \frac{\Gamma(-t) \Gamma\left(\frac{1}{2}+a^{\prime}+\beta+t\right) \Gamma\left(\frac{1}{2}-b^{\prime}+\beta+t\right)}{\Gamma(1+2 \beta+t)}\left(\frac{z}{1-z}\right)^{\frac{1}{2}+\beta+a^{\prime}+t} .
\end{aligned}
$$

As an intermediate step, we rewrite $K_{\text {split }}$ as a Mellin-Barnes integral:

$$
\begin{aligned}
K_{\mathrm{split}}\left(\alpha, \beta \mid h_{i}\right)= & \frac{\Gamma\left(1-h_{12}+h_{34}\right)}{\Gamma\left(1+h_{14}+h_{23}\right)} \frac{2 \beta}{\Gamma\left(\frac{1}{2}-h_{12} \pm \alpha\right)} \frac{\Gamma\left(\frac{1}{2}+h_{23}+\beta\right)}{\Gamma\left(\frac{1}{2}-h_{23}+\beta\right)} \int[d s] \frac{\Gamma(-s) \Gamma\left(\frac{1}{2}-h_{12}+s \pm \alpha\right)}{\Gamma\left(1-h_{12}+h_{34}+s\right)} \\
& \times \frac{\Gamma\left(2 h_{1}-1-s\right) \Gamma\left(h_{12}+h_{3}+h_{4}-1-s\right) \Gamma\left(\frac{3}{2}-h_{1}-h_{4}+\beta+s\right)}{\Gamma\left(h_{1}+h_{4}-\frac{1}{2}+\beta-s\right)}
\end{aligned}
$$

Closing the contour to the left ${ }^{17}$ and picking up poles at $s=-\mathbb{N}, s= \pm \alpha-\frac{1}{2}+h_{12}-\mathbb{N}$, we obtain the following closed-form formula for $K_{\text {split }}$ :

$$
K_{\text {split }}\left(\alpha, \beta \mid h_{1}, h_{2}, h_{3}, h_{4}\right)=I_{1}\left(\alpha, \beta \mid h_{i}\right)+I_{2}\left(\alpha, \beta \mid h_{i}\right)+I_{2}\left(-\alpha, \beta \mid h_{i}\right)
$$

\footnotetext{
${ }^{16}$ Note that the poles of $K_{0}\left(\alpha, \beta \mid h_{\phi}\right)$ in $\beta$ are related to the poles in $\alpha$ through eq. (3.14). In particular, the $\beta$ residues are Wilson polynomials in $\alpha$.

${ }^{17}$ Closing the contour to the right would mean picking up poles at $s=2 h_{1}-1+\mathbb{N}$ and $s=h_{12}+h_{3}+$ $h_{4}-1+\mathbb{N}$. In the case of equal external dimensions, these two series of poles collide to form a single series of double poles.
} 
where

$$
\left.\begin{array}{rl}
I_{1}\left(\alpha, \beta \mid h_{i}\right)= & \frac{\Gamma\left(1-h_{12}+h_{34}\right)}{\Gamma\left(1+h_{14}+h_{23}\right)} \frac{2 \beta}{S\left(h_{2}+h_{4}+\alpha-\beta\right) S\left(h_{2}+h_{4}-\alpha-\beta\right)} \\
& \times \frac{\Gamma\left(\frac{1}{2}+h_{14}+\beta\right) \Gamma\left(\frac{1}{2}+h_{23}+\beta\right) \Gamma\left(\frac{3}{2}-h_{1}-h_{4}+\beta\right)}{\Gamma\left(\frac{1}{2}-h_{12}+\alpha\right) \Gamma\left(h_{2}+h_{3}-\frac{1}{2}-\beta\right)} \\
& \times{ }_{4} \tilde{F}_{3}\left[\begin{array}{c}
\frac{1}{2}+h_{14}+\beta, \frac{1}{2}-h_{23}+\beta, \frac{3}{2}-h_{1}-h_{4}+\beta, \frac{3}{2}-h_{2}-h_{3}+\beta \\
1+2 \beta, 2-h_{2}-h_{4}+\alpha+\beta, 2-h_{2}-h_{4}-\alpha+\beta
\end{array} ; 1\right], \\
I_{2}\left(\alpha, \beta \mid h_{i}\right)= & -\frac{\Gamma\left(1-h_{12}+h_{34}\right)}{\Gamma\left(1+h_{14}+h_{23}\right)} \frac{2 \beta}{S\left(h_{2}+h_{4}+\alpha-\beta\right)} \\
& \times \frac{\Gamma\left(h_{1}+h_{2}-\frac{1}{2}+\alpha\right) \Gamma\left(h_{3}+h_{4}-\frac{1}{2}+\alpha\right)}{S(2 \alpha) \Gamma\left(\frac{1}{2}-h_{12}-\alpha\right) \Gamma\left(\frac{1}{2}+h_{34}-\alpha\right)} \frac{\Gamma\left(\frac{1}{2}+h_{23}+\beta\right)}{\Gamma\left(\frac{1}{2}-h_{23}+\beta\right)} \\
& \times{ }_{4} \tilde{F}_{3}\left[\frac{1}{2}-h_{12}+\alpha, \frac{1}{2}-h_{34}+\alpha, h_{1}+h_{2}-\frac{1}{2}+\alpha, h_{3}+h_{4}-\frac{1}{2}+\alpha\right. \\
1+2 \alpha, h_{2}+h_{4}+\alpha+\beta, h_{2}+h_{4}+\alpha-\beta
\end{array}\right] .
$$

Here we used the notation $C(x)=\cos (\pi x) / \pi, S(x)=\sin (\pi x) / \pi$, and the ${ }_{4} \tilde{F}_{3}(1)$ are regularized hypergeometric functions. In passing we remark that the hypergeometric functions appearing in (4.11) are balanced, ${ }^{18}$ which implies that they obey various interesting properties. In particular, $I_{1}$ and $I_{2}$ can be rewritten using three-term contiguous relations [41].

Above we claimed that $K_{\text {split }}\left(\alpha, \beta \mid h_{i}\right)$ was analytic in $\beta$ on the right half plane. This is not completely manifest from the expressions in eq. (4.11); in fact, it appears that both $I_{1}$ and $I_{2}$ have singularities at $\beta=h_{2}+h_{4} \pm \alpha+\mathbb{N}$. However, it can be shown (using hypergeometric identities, see e.g. [41]) that the residues in $I_{1}(\alpha, \beta)$ and $I_{2}( \pm \alpha, \beta)$ at these points exactly cancel. Equivalently, analyticity follows from a contour pinching argument applied to the Mellin-Barnes integral in eq. (4.9).

In passing, we claim that $K_{\text {split }}$ has the following symmetry:

$$
K_{\text {split }}\left(\alpha, \beta \mid h_{1}, h_{2}, h_{3}, h_{4}\right)=K_{\text {split }}\left(\alpha, \beta \mid h_{3}^{\natural}, h_{4}^{\natural}, h_{1}^{\natural}, h_{2}^{\natural}\right)
$$

cf. eq. (3.50a) for the normal kernel. ${ }^{19}$ To establish (4.12), one develops an alternate Mellin-Barnes representation for $K_{\text {split }}$, by changing the order of integration:

$$
\begin{aligned}
& K_{\text {split }}\left(\alpha, \beta \mid h_{i}\right)=\frac{\Gamma\left(1-h_{12}+h_{34}\right)}{\Gamma\left(1+h_{14}+h_{23}\right)} \frac{2 \beta}{\Gamma\left(\frac{1}{2}+h_{34} \pm \alpha\right)} \frac{\Gamma\left(\frac{1}{2}+h_{14}+\beta\right)}{\Gamma\left(\frac{1}{2}-h_{14}+\beta\right)} \\
& \times \int[d t] \frac{\Gamma(-t) \Gamma\left(\frac{1}{2}+a^{\prime}+\beta+t\right) \Gamma\left(\frac{1}{2}-b^{\prime}+\beta+t\right)}{\Gamma(1+2 \beta+t)} \frac{\Gamma\left(h_{1}+h_{3}-1-\beta-t \pm \alpha\right) \Gamma\left(\frac{3}{2}-h_{1}-h_{4}+\beta+t\right)}{\Gamma\left(h_{2}+h_{3}-\frac{1}{2}-\beta-t\right)} .
\end{aligned}
$$

Closing the contour to the right, we find a representation of $K_{\text {split }}$ of the schematic form (4.10), with $I_{1,2}\left(\alpha, \beta \mid h_{i}\right)$ replaced by functions $J_{1,2}\left(\alpha, \beta \mid h_{i}\right)$ obeying

$$
I_{k}\left(\alpha, \beta \mid h_{1}, h_{2}, h_{3}, h_{4}\right)=J_{k}\left(\alpha, \beta \mid h_{3}^{\natural}, h_{4}^{\natural}, h_{1}^{\natural}, h_{2}^{\natural}\right), \quad k=1,2 .
$$

\footnotetext{
${ }^{18} \mathrm{~A}$ hypergeometric function ${ }_{p} F_{q}\left(a_{1}, \ldots, a_{p} ; b_{1}, \ldots, b_{q} ; z\right)$ is said to be balanced or Saalschützian if $\sum_{i=1}^{p} a_{i}-\sum_{j=1}^{q} b_{j}=-1$.

${ }^{19}$ We also note the existence of a rather mysterious relation between $I_{1}\left(\alpha, \beta \mid h_{i}\right)$ and $I_{2}\left(\alpha, \beta \mid h_{i}\right)$, namely $I_{2}\left(\alpha, \beta \mid h_{1}, h_{2}, h_{3}, h_{4}\right)=\frac{N_{s}(\alpha)}{N_{t}(\beta)} \frac{C\left(\beta-h_{23}\right) S\left(h_{2}+h_{4}+\alpha+\beta\right)}{C\left(\alpha+h_{3}+h_{4}\right) S(2 \beta)} I_{1}\left(\beta, \alpha \mid 1-h_{1}, 1-h_{4}, 1-h_{3}, 1-h_{2}\right)$.
} 
This proves eq. (4.12).

Let us finally return to eq. (4.7). The modified falloff of the split kernel allows one to close the contour in the right $\beta$ plane and pick up the poles, which we have just demonstrated can only come from $F(\beta)$. Therefore, up to simple numerical factor the split kernel considered as a function of $\alpha$ for a fixed $\beta$ is precisely the $s$-channel alpha space transform of a single $t$-channel conformal block. It is therefore of interest to consider the analytic properties of $K_{\text {split }}\left(\alpha, \beta \mid h_{i}\right)$ in $\alpha$ as well. For example, for identical external dimensions $h_{i}$ a contour pinching argument applied to eq. (4.9) shows that $K_{\text {split }}\left(\alpha, \beta \mid h_{\phi}\right)$ has double rather than single poles at the double-trace values $\alpha= \pm\left(2 h_{\phi}-\frac{1}{2}+\mathbb{N}\right)$, reflecting the logarithmic behavior of the $k_{\beta+1 / 2}^{s}(z)$ as $z \rightarrow 1$ in position space. This most clearly demonstrates the impossibility of expressing physical conformal blocks in one channel as proper sums of blocks in the crossed channel and consequently the necessity of using a different basis of functions like our $\Psi_{\alpha}(z)$ to arrive at a meaningful crossing symmetry kernel.

\subsection{Using the $\xi_{n}$ as a basis}

It appears that a special role is played by the alpha space functions $\xi_{n}^{s}\left(\alpha \mid h_{i}\right), \xi_{n}^{t}\left(\alpha \mid h_{i}\right)$ and $\xi_{n}^{0}\left(\alpha \mid h_{\phi}\right)$, defined in eqs. (3.32), (3.34), (3.39). In fact, these basis functions furnish infinitely many solutions to crossing symmetry. To make this concrete, consider the mixed-correlator bootstrap equation (3.10), which is automatically solved if $F_{s, t}(\alpha)$ are chosen as follows:

$$
F_{s}(\alpha)=\sum_{n \text { even }} c_{n} \xi_{n}^{s}\left(\alpha \mid h_{i}\right), \quad F_{t}(\alpha)=\sum_{n \text { even }} c_{n} \xi_{n}^{t}\left(\alpha \mid h_{i}\right)
$$

It is crucial that the same coefficients $c_{n}$ appear both in $F_{s}(\alpha)$ and $F_{t}(\alpha)$, and that only $\xi_{n}$ with even $n$ appear. The reason is that the $\xi_{n}^{s, t}(\alpha)$ with odd $n$ are antisymmetric under crossing. To understand this more intuitively, it is instructive to analyze the $\xi_{n}$ in position space. Using eq. (2.29), we find that the $z$-space versions of $\xi_{n}^{s}\left(\alpha \mid h_{i}\right)$ and $\xi_{n}^{t}\left(\alpha \mid h_{i}\right)$ are given by

$$
\left\{\begin{array}{l}
\xi_{n}^{s}\left(z \mid h_{i}\right) \\
\xi_{n}^{t}\left(z \mid h_{i}\right)
\end{array}\right\}=n ! \Gamma\left(2 h_{2}+n\right) \Gamma\left(h_{1}+h_{3}+h_{24}+n\right) z^{2 h_{2}}\left\{\begin{array}{l}
P_{n}^{\left(a+b, a^{\prime}+b^{\prime}\right)}(1-2 z) \\
P_{n}^{\left(a^{\prime}+b^{\prime}, a+b\right)}(1-2 z)
\end{array}\right\} .
$$

Given eq. (4.16), it follows directly that

$$
\xi_{n}^{s}\left(z \mid h_{i}\right)=(-1)^{n}\left(\frac{z}{1-z}\right)^{2 h_{2}} \xi_{n}^{t}\left(1-z \mid h_{i}\right)
$$

where we use that $P_{n}^{(p, q)}(-x)=(-1)^{n} P_{n}^{(q, p)}(x)$. Comparing to the crossing equation (2.82), one confirms that the $\xi_{n}$ with even (resp. odd) $n$ are symmetric (resp. antisymmetric) under crossing symmetry.

Next, we will consider the CB decomposition of the functions $\xi_{n}$, at least schematically. Notice that $\xi_{n}^{s}\left(\alpha \mid h_{i}\right)$ only has poles at $\alpha=h_{1}+h_{2}-\frac{1}{2}+\mathbb{N}$, as well as mirror poles on the left half plane. Given our discussion in section 2 , this implies that $\xi_{n}^{s}\left(\alpha \mid h_{i}\right)$ has a CB decomposition consisting of operators of dimensions $h_{1}+h_{2}+\mathbb{N}$. Such a conformal block decomposition looks similar to a mean-field solution, where only double-twist primaries 
$\left[\phi_{1} \phi_{2}\right]_{n} \sim \phi_{1} \stackrel{\leftrightarrow}{\partial^{n}} \phi_{2}$ contribute. Similarly, $\xi_{n}^{t}$ has a CB decomposition with a spectrum given by $h_{2}+h_{3}+\mathbb{N}$.

For definiteness, we will compute the $\mathrm{CB}$ decomposition of $\xi_{n}^{0}\left(\alpha \mid h_{\phi}\right)$ explicitly. The position-space version of $\xi_{n}^{0}\left(\alpha \mid h_{\phi}\right)$ is a limiting case of (4.16), namely

$$
\xi_{n}^{0}\left(z \mid h_{\phi}\right)=n ! \Gamma^{2}\left(2 h_{\phi}+n\right) z^{2 h_{\phi}} P_{n}(1-2 z)
$$

where $P_{n}$ denotes a Legendre polynomial. As above, these functions are crossing (anti)symmetric for even (odd) $n$, as follows from

$$
\xi_{n}^{0}\left(z \mid h_{\phi}\right)=(-1)^{n}\left(\frac{z}{1-z}\right)^{2 h_{\phi}} \xi_{n}^{0}\left(1-z \mid h_{\phi}\right) .
$$

The CB decomposition of $\xi_{n}^{0}\left(z \mid h_{\phi}\right)$ can be found using alpha space technology; in particular, its residues in alpha space are equal to Wilson polynomials evaluated at certain values of $\alpha$. The precise result is

$$
\xi_{n}^{0}\left(z \mid h_{\phi}\right)=\sum_{m=0}^{\infty} A_{m}^{(n)} k_{2 h_{\phi}+m}(z)
$$

where

$$
A_{m}^{(n)}=\Gamma^{2}\left(2 h_{\phi}+n\right) n ! \frac{(-1)^{m}}{m !} \frac{\left(2 h_{\phi}\right)_{m}^{2}}{\left(4 h_{\phi}-1+m\right)_{m}}{ }_{4} F_{3}\left(\begin{array}{c}
-n,-m, n+1,4 h_{\phi}-1+m \\
2 h_{\phi}, 2 h_{\phi}, 1
\end{array} ;\right)
$$

Notice that the coefficients $A_{m}^{(n)}$ are sign-alternating: $\operatorname{sgn}\left(A_{m}^{(n)}\right)=(-1)^{m}$, provided that $h_{\phi}>0$. This implies that the $\xi_{n}$ do not correspond to unitary solutions of crossing.

At least formally, it is possible to derive selection rules for alpha space densities using the functions $\xi_{n}$. We will focus on the identical-operator case for simplicity. Recall that the $\xi_{n}^{0}$ form a basis of the Hilbert space $\mathcal{H}_{0}$ introduced in section 3.5. This implies that if a density $F(\alpha) \in \mathcal{H}_{0}$ is crossing symmetric, it must obey

$$
\int \frac{[d \alpha]}{\mathcal{M}_{0}\left(\alpha ; h_{\phi}\right)} \xi_{n}^{0}\left(\alpha \mid h_{\phi}\right) F(\alpha)=0 \quad \text { for } \quad n=1,3,5, \ldots
$$

This selection rule manifestly holds if $F(\alpha)$ is of the following form:

$$
F(\alpha)=\sum_{n \text { even }} c_{n} \xi_{n}^{0}\left(\alpha \mid h_{\phi}\right)
$$

cf. eq. (4.15). Of course, requiring that $F(\alpha)$ is normalizable imposes constraints on the growth of the coefficients $c_{n}$ as $n \rightarrow \infty$.

Unfortunately an alpha space density of the form (4.23) cannot belong to an interacting CFT: it would have a CB decomposition with exchanged operators of dimensions $2 h_{\phi}+\mathbb{N}$ and nothing else - in particular, requiring that $F(\alpha) \in \mathcal{H}_{0}$ rules out an identity operator contribution. These unphysical constraints on the spectrum of $F$ are very similar to the issue encountered in section 4.1. We also stress that (4.23) generically corresponds to a nonunitary $\mathrm{CB}$ decomposition, in line with our remarks below eq. (4.20). Imposing unitarity 
leads to additional constraints on the coefficients $c_{n}$, and in future work it would certainly be interesting to examine these in detail.

To better understand the role played by the $\xi_{n}$, we will briefly consider how these ideas apply to a mean-field correlator:

$$
F_{\mathrm{MFT}}(z)=t_{1} F_{1}(z)+t_{2} F_{2}(z)
$$

with

$$
F_{1}(z)=z^{2 h_{\phi}} \quad \text { and } \quad F_{2}(z)=1+\left(\frac{z}{1-z}\right)^{2 h_{\phi}} .
$$

Both pieces $F_{1,2}$ are crossing symmetric by themselves, but only their combination with $t_{2} \pm t_{1} \geq 0$ is unitary. This follows from the CB decompositions (2.56) and (2.59). ${ }^{20}$ Separately, $F_{1}$ and $F_{2}$ contain contributions from an infinite tower of operators of dimension $2 h_{\phi}+n$, but the contributions for odd (resp. even) $n$ cancel out when $t_{1}=t_{2}$ (resp. $\left.t_{1}=-t_{2}\right)$. The combinations with $t_{1}= \pm t_{2}$ correspond to generalized free fields with bosonic (resp. fermionic) statistics.

Can we decompose $F_{1}$ and $F_{2}$ à la eq. (4.23)? As for $F_{1}$, we see by inspection that

$$
F_{1}(z)=\frac{1}{\Gamma^{2}\left(2 h_{\phi}\right)} \xi_{0}^{0}\left(z \mid h_{\phi}\right)
$$

consistent with the fact that $F_{1}$ is crossing symmetric and non-unitary. In particular, this shows that $F_{1}(\alpha) \in \mathcal{H}$. Notice that this is only possible because $F_{1}(z)$ has no unit operator contribution. Since $F_{2}(z)$ does have a unit operator contribution, it follows that $F_{2}(z)$ cannot be decomposed as in eq. (4.23). Nevertheless, we compute

$$
\left(\frac{z}{1-z}\right)^{2 h_{\phi}}=\sum_{n=0}^{\infty} f_{n} \xi_{n}^{0}\left(z \mid h_{\phi}\right), \quad f_{n}=\frac{1}{\Gamma^{2}\left(2 h_{\phi}\right)} \frac{1+2 n}{n !\left(1-2 h_{\phi}+n\right)} \frac{1}{\left(2 h_{\phi}-n\right)_{2 n}} .
$$

Strictly speaking this holds only for $h_{\phi}<1 / 2$; for generic $h_{\phi}$, (4.27) makes sense only after analytic continuation. Notice that (4.27) contains terms with both even and odd $n$. This is consistent with the fact that $[z /(1-z)]^{2 h_{\phi}}$ by itself has no definite crossing behaviour. Another interesting feature is that the $f_{n}$ are not sign-definite; in fact, $\operatorname{sgn}\left(f_{n}\right)=(-1)^{n}$ provided that $h_{\phi}<1 / 2$. However, we know from eq. (2.59) that $[z /(1-z)]^{2 h_{\phi}}$ has a CB decomposition with positive coefficients. We conclude that there is a conspiracy between the coefficients $f_{n}$ from eq. (4.27) and the $A_{m}^{(n)}$ from (4.20) that guarantees that the full CB decomposition is unitary.

The above example shows how the idea to draw selection rules from the $\xi_{n}$ runs into problems when naively applied to CFT correlators. Nonetheless, it may be true that a modified version of eq. (4.22) holds after carefully regulating the identity operator contribution. We leave this question for future work.

\footnotetext{
${ }^{20}$ Here we are interested in the case $p=q$ of eq. (2.59), which reads

$$
\left(\frac{z}{1-z}\right)^{p}=\sum_{n=0}^{\infty} \frac{(p)_{n}^{2}}{n !(2 p-1+n)_{n}} k_{p+n}(z) .
$$
}




\section{Discussion}

This paper has outlined how Sturm-Liouville theory provides a framework to study CFTs. Inspired by classic results [73], we discussed the decomposition of a CFT four-point correlator in terms of a new basis of functions $\Psi_{\alpha}(z)$ and explained how the familiar conformal block decomposition can be obtained by analytic continuation in $\alpha$. The alpha space decomposition allowed us to formulate crossing symmetry in terms of an eigenfunction problem for some integral kernels: in particular equation (4.1) is a mathematically precise version of the abstract idea expressed by equation (1.11) in the introduction. It features an explicitly known crossing symmetry kernel $K_{0}\left(\alpha, \beta \mid h_{\phi}\right)$ whose properties we analyzed in some detail.

In this paper we did not touch on the profound connection between the alpha space construction and the representation theory of the conformal group. Roughly speaking the dictionary is well-known: three-point functions map to Clebsch-Gordan kernels, conformal blocks are their square - as used in three-fold tensor products - and the crossing symmetry kernel is equal to a $6-j$ symbol for the conformal group. Moreover, the alpha space decomposition ought to correspond to tensor product decomposition into a direct integral over the principal unitary series of representations. We can however only make all these relations precise if we have a detailed knowledge of both the groups, the representations under consideration, and the Hilbert space of functions on which they act. ${ }^{21}$ For the case at hand the question appears to be partially solved in [68], which showed that the Wilson functions $\mathrm{W}_{\alpha}(\beta ; a, b, c, d)$ indeed appear as $6-j$ symbols for representations of the $\mathfrak{s l}(2, \mathbb{R})$ conformal algebra. Surprisingly this connection works provided three of the four external dimensions transform in the discrete unitary series, in contrast with the older discussion of [73] which is based entirely on the principal unitary series. ${ }^{22}$ It would be interesting to build on the results of [68] to explicitly connect all the dots between alpha space, one-dimensional unitary CFTs and representation theory. We hope to return to this problem in the near future.

It is of clear interest to generalize our analysis to $d \geq 2$ dimensions. This requires solving the Sturm-Liouville problem for the $d$-dimensional Casimir [77] on the square $(0,1) \times(0,1)$, or alternatively one could relate this kernel to a suitable set of $6-j$ symbols of the universal cover of $\mathrm{SO}(d, 2)$. The higher- $d$ alpha space picture will necessarily be more complicated, because both external and exchanged operators in higher- $d$ CFTs can carry a nontrivial Lorentz spin. An obvious generalization pertains to superconformal field theories in various $d$ [78]. Sturm-Liouville theory should also apply beyond four-point correlators in CFTs on $\mathbb{R}^{d}$; for instance, one can consider its application to CFTs in the presence of boundaries or defects.

Most of these problems are rather formal and group-theoretical in nature. In the framework of the conformal bootstrap, it is more exciting to investigate whether alpha space crossing equations can be leveraged to constrain CFT data, or - more ambitiously

\footnotetext{
${ }^{21}$ In this context it is important to note that the representations are only unitary in Lorentzian signature. In that case the conformal group is actually the universal cover of $\operatorname{SL}(2, \mathbb{R})[74]$, which has a richer class of inequivalent unitary representations [75] (see also [76] for a detailed discussion of the $4 d$ case).

${ }^{22}$ This is related to our basis functions being different form the usual shadow-symmetric blocks of [59] which are in fact the correct squared Clebsch-Gordan coefficients for three unitary principal series.
} 
- to solve bootstrap equations analytically. ${ }^{23}$ In section 4 we discussed some tentative ideas in this direction. Together with recent developments in the realm of Mellin space and the lightcone bootstrap, we are optimistic that alpha space can become part of the analytic bootstrap toolkit.

\section{Acknowledgments}

This work originated from discussions with Leonardo Rastelli and Pedro Liendo in Stony Brook in 2011, and we would like to thank them for their valuable contributions in these early stages. Moreover, we gratefully acknowledge discussions with the participants of the 'Back to the Bootstrap II' meeting in 2012 where an initial version of this work was first presented. We would like to thank Christopher Beem, Jyoti Bhattacharya, Liam Fitzpatrick, Simon Caron-Huot, Abhijit Gadde, Leszek Hadasz, Christoph Keller, Zohar Komargodski, Hugh Osborn, Slava Rychkov, Volker Schomerus, David Simmons-Duffin and Sasha Zhiboedov for more recent discussions and/or comments. This research was supported in part by Perimeter Institute for Theoretical Physics. Research at Perimeter Institute is supported by the Government of Canada through Industry Canada and by the Province of Ontario through the Ministry of Research and Innovation. This work was additionally supported by a grant from the Simons Foundation (\#488659).

\section{A Computing the inner product $\left\langle\Psi_{\alpha}, \Psi_{\beta}\right\rangle$}

In this section, we will prove eq. (2.15) by computing the inner product $\left\langle\Psi_{\alpha}, \Psi_{\beta}\right\rangle$, as defined in eq. (2.7). Concretely, we must perform the following integral:

$$
\left\langle\Psi_{\alpha}, \Psi_{\beta}\right\rangle=\int_{0}^{1} \frac{d z}{z^{2}} \Psi_{\alpha}(z) \Psi_{\beta}(z)
$$

where we used that $\overline{\Psi_{\alpha}(z)}=\Psi_{\alpha}(z)$ for imaginary $\alpha$. As a first step, we write $\Psi_{\alpha}(z)$ and $\Psi_{\beta}(z)$ using a Mellin-Barnes representation:

$$
\Psi_{\alpha}(z)=\frac{1}{\Gamma\left(\frac{1}{2} \pm \alpha\right)} \int[d s] \frac{\Gamma(-s) \Gamma\left(\frac{1}{2}+s \pm \alpha\right)}{\Gamma(1+s)}\left(\frac{1-z}{z}\right)^{s}
$$

Naively, the $z$-integral (A.1) is logarithmically divergent, the divergence coming from the region near $z=0$. To resolve this divergence, we regulate $\Psi_{\beta}(z)$ by writing it as follows:

$$
\Psi_{\beta}(z) \rightarrow z_{2}^{\epsilon} F_{1}\left(\begin{array}{c}
\frac{1}{2}+\beta, \frac{1}{2}-\beta \\
1+\epsilon
\end{array} ;-\frac{1-z}{z}\right)=z^{\epsilon} \frac{\Gamma(1+\epsilon)}{\Gamma\left(\frac{1}{2} \pm \beta\right)} \int[d t] \frac{\Gamma(-t) \Gamma\left(\frac{1}{2}+t \pm \beta\right)}{\Gamma(1+\epsilon+t)}\left(\frac{1-z}{z}\right)^{t}
$$

for $\epsilon>0$. This behaves as $O\left(z^{1 / 2+\epsilon}\right)$ at small $z$. Evidently, in the limit $\epsilon \rightarrow 0$, the above function reduces to $\Psi_{\beta}(z)$.

\footnotetext{
${ }^{23}$ See [79] for a connection between the conformal Casimir and integrability, which may be helpful in this context.
} 
At this point, the inner product eq. (A.1) is given by triple integral, schematically

$$
\left\langle\Psi_{\alpha}, \Psi_{\beta}\right\rangle=\int_{0}^{1} d z \int[d s] \int[d t](\ldots) .
$$

Since we have regulated the integrand, this integral converges and we can exchange the order of the different integrals. We do the $z$-integral first, which is a simple beta function integral. The result is

$$
\begin{aligned}
\ldots=\frac{\Gamma(1+\epsilon)}{\Gamma\left(\frac{1}{2} \pm \alpha\right) \Gamma\left(\frac{1}{2} \pm \beta\right)} \int[d s] \frac{\Gamma(-s) \Gamma\left(\frac{1}{2} \pm s+\alpha\right)}{\Gamma(1+s)} \\
\times \int[d t] \frac{\Gamma(-t) \Gamma\left(\frac{1}{2}+t \pm \beta\right)}{\Gamma(1+\epsilon+t)} \frac{\Gamma(1+s+t) \Gamma(-1-s-t+\epsilon)}{\Gamma(\epsilon)} .
\end{aligned}
$$

We now do the $t$-integral, using the second Barnes lemma. This yields

$$
\left\langle\Psi_{\alpha}, \Psi_{\beta}\right\rangle=\lim _{\epsilon \rightarrow 0} \frac{\Gamma(1+\epsilon)}{\Gamma\left(\frac{1}{2} \pm \alpha\right) \Gamma\left(\frac{1}{2}+\epsilon \pm \beta\right)} \int[d s] \frac{\Gamma(-s) \Gamma\left(\frac{1}{2} \pm s+\alpha\right) \Gamma\left(-\frac{1}{2}-s+\epsilon \pm \beta\right)}{\Gamma(-s+\epsilon)} .
$$

At this stage we can take the limit $\epsilon \rightarrow 0$ everywhere, except in the two factors $\Gamma\left(-\frac{1}{2}-\right.$ $s+\epsilon \pm \beta)$ :

$$
\ldots=\frac{1}{\Gamma\left(\frac{1}{2} \pm \alpha\right) \Gamma\left(\frac{1}{2} \pm \beta\right)} \int[d s] \Gamma\left(\frac{1}{2}+s \pm \alpha\right) \Gamma\left(-\frac{1}{2}-s+\epsilon \pm \beta\right) .
$$

This integral can be computed using the first Barnes lemma, yielding

$$
\begin{aligned}
\left\langle\Psi_{\alpha}, \Psi_{\beta}\right\rangle & =\frac{1}{\Gamma\left(\frac{1}{2} \pm \alpha\right) \Gamma\left(\frac{1}{2} \pm \beta\right)} \lim _{\epsilon \rightarrow 0} Z_{\epsilon}(\alpha, \beta), \\
Z_{\epsilon}(\alpha, \beta) & =\frac{1}{\Gamma(2 \epsilon)} \Gamma(\alpha+\beta+\epsilon) \Gamma(\alpha-\beta+\epsilon) \Gamma(-\alpha+\beta+\epsilon) \Gamma(-\alpha-\beta+\epsilon) .
\end{aligned}
$$

To conclude, we need to analyze the limit $\epsilon \rightarrow 0$ of $Z_{\epsilon}(\alpha, \beta)$, which we claim is the sum of two Dirac delta functions:

$$
\lim _{\epsilon \rightarrow 0} \int[d \beta] Z_{\epsilon}(\alpha, \beta) f(\beta)=\Gamma( \pm 2 \alpha)[f(\alpha)+f(-\alpha)],
$$

where $f(\alpha)$ is a test function. Notice that eq. (A.9) is sufficient to establish eq. (2.15), after remarking that

$$
\frac{2 \Gamma( \pm 2 \alpha)}{\Gamma\left(\frac{1}{2} \pm \alpha\right)^{2}}=N(\alpha)
$$

The proof of (A.9) goes as follows. We start by noticing that $\lim _{\epsilon \rightarrow 0} Z_{\epsilon}(\alpha, \beta)$ vanishes, unless $\beta= \pm \alpha \pm n$ for some integer $n$. If $n \neq 0$, the limit $\epsilon \rightarrow 0$ is finite, hence such points do not contribute to the integral in eq. (A.9). Hence it suffices to consider the cases $\beta=\alpha$ and $\beta=-\alpha$. For concreteness, let's consider $\beta=\alpha$, in which case we can approximate $Z_{\epsilon}(\alpha, \beta)$ by

$$
Z_{\epsilon}(\alpha, \beta) \underset{\beta \rightarrow \alpha}{\sim} \Gamma( \pm 2 \alpha) \omega_{\epsilon}(\alpha-\beta), \quad \omega_{\epsilon}(\alpha)=\frac{\Gamma(\epsilon \pm \alpha)}{\Gamma(2 \epsilon)}
$$


It is straightforward to see that $\omega_{\epsilon}(\alpha)$ behaves as a delta function along the imaginary axis, i.e.

$$
\lim _{\epsilon \rightarrow 0} \int[d \alpha] \omega_{\epsilon}(\alpha) f(\alpha)=f(0) .
$$

This follows from the fact that $\omega_{\epsilon}(\alpha)$ is peaked around $\alpha=0$ with width $\epsilon$ (taking $\alpha$ to be imaginary) together with the fact that

$$
\int[d \alpha] \omega_{\epsilon}(\alpha)=\frac{1}{4^{\epsilon}} \rightarrow 1
$$

The same argument holds for the region where $\beta=-\alpha$. This allows us to conclude.

Open Access. This article is distributed under the terms of the Creative Commons Attribution License (CC-BY 4.0), which permits any use, distribution and reproduction in any medium, provided the original author(s) and source are credited.

\section{References}

[1] S. Ferrara, A.F. Grillo, G. Parisi and R. Gatto, Covariant expansion of the conformal four-point function, Nucl. Phys. B 49 (1972) 77 [Erratum ibid. B 53 (1973) 643] [INSPIRE].

[2] S. Ferrara, A.F. Grillo and R. Gatto, Tensor representations of conformal algebra and conformally covariant operator product expansion, Annals Phys. 76 (1973) 161 [inSPIRE].

[3] A.M. Polyakov, Nonhamiltonian approach to conformal quantum field theory, Zh. Eksp. Teor. Fiz. 66 (1974) 23 [Sov. Phys. JETP 39 (1974) 9] [INSPIRE].

[4] A.A. Belavin, A.M. Polyakov and A.B. Zamolodchikov, Infinite conformal symmetry in two-dimensional quantum field theory, Nucl. Phys. B 241 (1984) 333 [INSPIRE].

[5] R. Rattazzi, V.S. Rychkov, E. Tonni and A. Vichi, Bounding scalar operator dimensions in $4 D$ CFT, JHEP 12 (2008) 031 [arXiv:0807.0004] [INSPIRE].

[6] I. Heemskerk, J. Penedones, J. Polchinski and J. Sully, Holography from conformal field theory, JHEP 10 (2009) 079 [arXiv: 0907.0151] [INSPIRE].

[7] R. Rattazzi, S. Rychkov and A. Vichi, Bounds in $4 D$ conformal field theories with global symmetry, J. Phys. A 44 (2011) 035402 [arXiv: 1009.5985] [INSPIRE].

[8] D. Poland, D. Simmons-Duffin and A. Vichi, Carving out the space of 4D CFTs, JHEP 05 (2012) 110 [arXiv:1109.5176] [INSPIRE].

[9] S. El-Showk, M.F. Paulos, D. Poland, S. Rychkov, D. Simmons-Duffin and A. Vichi, Solving the $3 D$ Ising model with the conformal bootstrap, Phys. Rev. D 86 (2012) 025022 [arXiv: 1203.6064] [INSPIRE].

[10] D. Pappadopulo, S. Rychkov, J. Espin and R. Rattazzi, OPE convergence in conformal field theory, Phys. Rev. D 86 (2012) 105043 [arXiv: 1208.6449] [INSPIRE].

[11] S. El-Showk and M.F. Paulos, Bootstrapping conformal field theories with the extremal functional method, Phys. Rev. Lett. 111 (2013) 241601 [arXiv:1211.2810] [INSPIRE].

[12] A.L. Fitzpatrick, J. Kaplan, D. Poland and D. Simmons-Duffin, The analytic bootstrap and AdS superhorizon locality, JHEP 12 (2013) 004 [arXiv:1212.3616] [INSPIRE]. 
[13] Z. Komargodski and A. Zhiboedov, Convexity and liberation at large spin, JHEP 11 (2013) 140 [arXiv: 1212.4103] [INSPIRE].

[14] F. Gliozzi, More constraining conformal bootstrap, Phys. Rev. Lett. 111 (2013) 161602 [arXiv:1307.3111] [INSPIRE].

[15] C. Beem, M. Lemos, P. Liendo, W. Peelaers, L. Rastelli and B.C. van Rees, Infinite chiral symmetry in four dimensions, Commun. Math. Phys. 336 (2015) 1359 [arXiv:1312.5344] [INSPIRE].

[16] S. El-Showk, M.F. Paulos, D. Poland, S. Rychkov, D. Simmons-Duffin and A. Vichi, Solving the $3 D$ Ising model with the conformal bootstrap II. c-minimization and precise critical exponents, J. Stat. Phys. 157 (2014) 869 [arXiv:1403.4545] [InSPIRE].

[17] F. Kos, D. Poland and D. Simmons-Duffin, Bootstrapping mixed correlators in the $3 D$ Ising model, JHEP 11 (2014) 109 [arXiv: 1406.4858] [INSPIRE].

[18] L.F. Alday and A. Zhiboedov, Conformal bootstrap with slightly broken higher spin symmetry, JHEP 06 (2016) 091 [arXiv:1506. 04659] [INSPIRE].

[19] T. Hartman, S. Jain and S. Kundu, Causality constraints in conformal field theory, JHEP 05 (2016) 099 [arXiv: 1509.00014] [INSPIRE].

[20] H. Kim, P. Kravchuk and H. Ooguri, Reflections on conformal spectra, JHEP 04 (2016) 184 [arXiv: 1510.08772] [INSPIRE].

[21] S.M. Chester and S.S. Pufu, Towards bootstrapping QED 3 , JHEP 08 (2016) 019 [arXiv: 1601.03476] [INSPIRE].

[22] P. Dey, A. Kaviraj and K. Sen, More on analytic bootstrap for $O(N)$ models, JHEP 06 (2016) 136 [arXiv: 1602.04928] [InSPIRE].

[23] D.M. Hofman, D. Li, D. Meltzer, D. Poland and F. Rejon-Barrera, A proof of the conformal collider bounds, JHEP 06 (2016) 111 [arXiv: 1603.03771] [INSPIRE].

[24] F. Kos, D. Poland, D. Simmons-Duffin and A. Vichi, Precision islands in the Ising and $O(N)$ models, JHEP 08 (2016) 036 [arXiv:1603.04436] [INSPIRE].

[25] S. El-Showk and M.F. Paulos, Extremal bootstrapping: go with the flow, arXiv:1605.08087 [INSPIRE].

[26] L.F. Alday, Large spin perturbation theory for conformal field theories, Phys. Rev. Lett. 119 (2017) 111601 [arXiv:1611.01500] [INSPIRE].

[27] D. Mazac, Analytic bounds and emergence of $A d S_{2}$ physics from the conformal bootstrap, JHEP 04 (2017) 146 [arXiv:1611.10060] [INSPIRE].

[28] D. Simmons-Duffin, The lightcone bootstrap and the spectrum of the $3 D$ Ising CFT, JHEP 03 (2017) 086 [arXiv: 1612.08471] [INSPIRE].

[29] S. Rychkov, EPFL lectures on conformal field theory in $D \geq 3$ dimensions, SpringerBriefs Phys., Springer, Cham Switzerland, (2016) [arXiv:1601.05000] [INSPIRE].

[30] J.D. Qualls, Lectures on conformal field theory, arXiv:1511.04074 [INSPIRE].

[31] D. Simmons-Duffin, The conformal bootstrap, in New frontiers in fields and strings, World Scientific, Singapore, (2017) [arXiv: 1602 .07982] [INSPIRE]. 
[32] G. Mack, D-independent representation of conformal field theories in D dimensions via transformation to auxiliary dual resonance models. Scalar amplitudes, arXiv:0907.2407 [INSPIRE].

[33] J. Penedones, Writing CFT correlation functions as AdS scattering amplitudes, JHEP 03 (2011) 025 [arXiv: 1011.1485] [INSPIRE].

[34] A.L. Fitzpatrick, J. Kaplan, J. Penedones, S. Raju and B.C. van Rees, A natural language for AdS/CFT correlators, JHEP 11 (2011) 095 [arXiv:1107.1499] [InSPIRE].

[35] A.L. Fitzpatrick and J. Kaplan, Analyticity and the holographic S-matrix, JHEP 10 (2012) 127 [arXiv: 1111.6972] [INSPIRE].

[36] A.L. Fitzpatrick and J. Kaplan, Unitarity and the holographic S-matrix, JHEP 10 (2012) 032 [arXiv:1112.4845] [INSPIRE].

[37] M.S. Costa, V. Goncalves and J. Penedones, Conformal Regge theory, JHEP 12 (2012) 091 [arXiv:1209.4355] [INSPIRE].

[38] V. Gonçalves, J. Penedones and E. Trevisani, Factorization of Mellin amplitudes, JHEP 10 (2015) 040 [arXiv:1410.4185] [INSPIRE].

[39] R. Gopakumar, A. Kaviraj, K. Sen and A. Sinha, Conformal bootstrap in Mellin space, Phys. Rev. Lett. 118 (2017) 081601 [arXiv:1609.00572] [InSPIRE].

[40] R. Gopakumar, A. Kaviraj, K. Sen and A. Sinha, A Mellin space approach to the conformal bootstrap, JHEP 05 (2017) 027 [arXiv:1611.08407] [INSPIRE].

[41] G.E. Andrews, R. Askey and R. Roy, Special functions, Cambridge University Press, Cambridge U.K., (1999).

[42] R. Koekoek and R.F. Swarttouw, The Askey-scheme of hypergeometric orthogonal polynomials and its q-analogue, math.CA/9602214.

[43] V.K. Dobrev, V.B. Petkova, S.G. Petrova and I.T. Todorov, Dynamical derivation of vacuum operator product expansion in Euclidean conformal quantum field theory, Phys. Rev. D 13 (1976) 887 [INSPIRE].

[44] B. Ponsot and J. Teschner, Liouville bootstrap via harmonic analysis on a noncompact quantum group, hep-th/9911110 [INSPIRE].

[45] B. Ponsot and J. Teschner, Clebsch-Gordan and Racah-Wigner coefficients for a continuous series of representations of $\mathcal{U}_{q}(\mathrm{SL}(2, R))$, Commun. Math. Phys. 224 (2001) 613 [math/0007097] [INSPIRE].

[46] L. Hadasz, M. Pawelkiewicz and V. Schomerus, Self-dual continuous series of representations for $\mathcal{U}_{q}(\operatorname{sl}(2))$ and $\mathcal{U}_{q}(\operatorname{osp}(1 \mid 2))$, JHEP 10 (2014) 091 [arXiv:1305.4596] [InSPIRE].

[47] M. Pawelkiewicz, V. Schomerus and P. Suchanek, The universal Racah-Wigner symbol for $\mathcal{U}_{q}(\operatorname{osp}(1 \mid 2))$, JHEP 04 (2014) 079 [arXiv:1307.6866] [INSPIRE].

[48] I. Esterlis, A.L. Fitzpatrick and D. Ramirez, Closure of the operator product expansion in the non-unitary bootstrap, JHEP 11 (2016) 030 [arXiv:1606.07458] [INSPIRE].

[49] G.W. Moore and N. Seiberg, Classical and quantum conformal field theory, Commun. Math. Phys. 123 (1989) 177 [INSPIRE].

[50] G.W. Moore and N. Seiberg, Lectures on RCFT, in Physics, geometry and topology, Springer, Boston MA U.S.A., (1989). 
[51] L. Alvarez-Gaumé, G. Sierra and C. Gomez, Topics in conformal field theory, CERN-TH-5540-89, CERN, Geneva Switzerland, (1989).

[52] A. Gadde, In search of conformal theories, arXiv:1702.07362 [INSPIRE].

[53] D. Gaiotto, D. Mazac and M.F. Paulos, Bootstrapping the 3D Ising twist defect, JHEP 03 (2014) 100 [arXiv:1310.5078] [INSPIRE].

[54] M. Billò, M. Caselle, D. Gaiotto, F. Gliozzi, M. Meineri and R. Pellegrini, Line defects in the 3D Ising model, JHEP 07 (2013) 055 [arXiv: 1304.4110] [INSPIRE].

[55] F. Gliozzi, P. Liendo, M. Meineri and A. Rago, Boundary and interface CFTs from the conformal bootstrap, JHEP 05 (2015) 036 [arXiv: 1502.07217] [INSPIRE].

[56] M. Billò, V. Gonçalves, E. Lauria and M. Meineri, Defects in conformal field theory, JHEP 04 (2016) 091 [arXiv: 1601. 02883] [INSPIRE].

[57] L.F. Alday and J.M. Maldacena, Comments on operators with large spin, JHEP 11 (2007) 019 [arXiv: 0708.0672] [INSPIRE].

[58] L.L. Littlejohn and A. Zettl, The Legendre equation and its self-adjoint operators, Electron. J. Diff. Equat. 2011 (2011) 1.

[59] F.A. Dolan and H. Osborn, Conformal four point functions and the operator product expansion, Nucl. Phys. B 599 (2001) 459 [hep-th/0011040] [InSPIRE].

[60] K. Symanzik, On calculations in conformal invariant field theories, Lett. Nuovo Cim. 3 (1972) 734 [INSPIRE].

[61] M. Flensted-Jensen and T.H. Koornwinder, The convolution structure for Jacobi function expansions, Ark. Mat. 11 (1973) 245.

[62] M. Flensted-Jensen and T.H. Koornwinder, Jacobi functions: the addition formula and the positivity of the dual convolution structure, Ark. Mat. 17 (1979) 139.

[63] T.H. Koornwinder, Jacobi functions and analysis on noncompact semisimple Lie groups, in Special functions: group theoretical aspects and applications, Springer, Dordrecht The Netherlands, (1984), pg. 1.

[64] T.H. Koornwinder, Special orthogonal polynomial systems mapped onto each other by the Fourier-Jacobi transform, in Polynômes Orthogonaux et Applications, Springer, Berlin Heidelberg Germany, (1985), pg. 174.

[65] J.A. Wilson, Some hypergeometric orthogonal polynomials, SIAM J. Math. Anal. 11 (1980) 690.

[66] S. Rychkov and P. Yvernay, Remarks on the convergence properties of the conformal block expansion, Phys. Lett. B 753 (2016) 682 [arXiv:1510.08486] [InSPIRE].

[67] W. Groenevelt, The Wilson function transform, Int. Math. Res. Not. 2003 (2003) 2779 [math.CA/0306424].

[68] W. Groenevelt, Wilson function transforms related to Racah coefficients, Acta Appl. Math. 91 (2006) 133 [math.CA/0501511].

[69] T. Regge, Symmetry properties of Racah's coefficients, Nuovo Cim. 11 (1959) 116 [INSPIRE].

[70] Ya.A. Granovskii and A.S. Zhedanov, Nature of the symmetry group of the 6j-symbol, Sov. Phys. JETP 67 (1988) 1982 [Zh. Eksp. Teor. Fiz. 94 (1988) 49]. 
[71] P.P. Boalch, Regge and Okamoto symmetries, Commun. Math. Phys. 276 (2007) 117 [math.RT/0603398].

[72] J. Fuchs and C. Schweigert, Symmetries, Lie algebras and representations, Cambridge University Press, Cambridge U.K., (2003) [InSPIRE].

[73] V.K. Dobrev, G. Mack, V.B. Petkova, S.G. Petrova and I.T. Todorov, Harmonic analysis on the $n$-dimensional Lorentz group and its application to conformal quantum field theory, in Harmonic analysis: on the $n$-dimensional Lorentz group and its application to conformal quantum field theory, Lect. Notes Phys. 63 (1977) 1 [INSPIRE].

[74] M. Lüscher and G. Mack, Global conformal invariance in quantum field theory, Commun. Math. Phys. 41 (1975) 203 [InSPIRE].

[75] L. Pukánszky, The Plancherel formula for the universal covering group of SL(R,2), Math. Ann. 156 (1964) 96.

[76] G. Mack, All unitary ray representations of the conformal group $\mathrm{SU}(2,2)$ with positive energy, Commun. Math. Phys. 55 (1977) 1 [INSPIRE].

[77] F.A. Dolan and H. Osborn, Conformal partial waves and the operator product expansion, Nucl. Phys. B 678 (2004) 491 [hep-th/0309180] [INSPIRE].

[78] C. Cordova, T.T. Dumitrescu and K. Intriligator, Multiplets of superconformal symmetry in diverse dimensions, arXiv:1612.00809 [INSPIRE].

[79] M. Isachenkov and V. Schomerus, Superintegrability of d-dimensional conformal blocks, Phys. Rev. Lett. 117 (2016) 071602 [arXiv:1602.01858] [INSPIRE]. 